\title{
Euphausiid Larvae in Plankton Samples from the Vicinity of the Antarctic Peninsula, February 1982
}

by Sigrid Marschall and Elke Mizdalski

Berichte zur Polarforschung Nr. 21/Februar 1985

Reports on Polar Research No. 21/February 1985 



\section{Contents}

paqe

Summary $-z$ usammenfassunq $\ldots \ldots \ldots \ldots \ldots \ldots \ldots \ldots$

1. Introduction $\ldots \ldots \ldots \ldots \ldots \ldots \ldots \ldots \ldots \ldots \ldots \ldots \ldots \ldots \ldots \ldots$

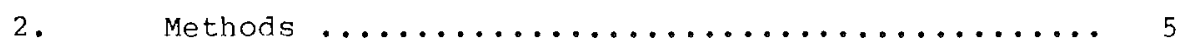

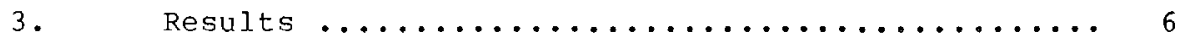

4. References $\ldots \ldots \ldots \ldots \ldots \ldots \ldots \ldots \ldots \ldots \ldots \ldots \ldots$

5. Station maps $\ldots \ldots \ldots \ldots \ldots \ldots \ldots \ldots \ldots \ldots \ldots \ldots$

6. Distribution maps $\ldots \ldots \ldots \ldots \ldots \ldots \ldots \ldots \ldots \ldots \ldots$

7. Euphausid larvae data lists .............. 18 


\section{Summary}

This report gives the results of the identification of euphausid larvae, caught during the Joint Biological Expedition on RRS "John Biscoe" in Eebruary 1982. Sampling was carried out with the RMT $1+8 \mathrm{~m}$ and the Nansen-Closina-Net in the region of the Antarctic Peninsula and the Scotia Sea. Larvae of four euphausid species occurred in this area. Euphausia superba was dominant.

\section{Zusammenfassung}

Im folgenden Bericht werden die Ergebnisse der Bestimmung und Stadieneinteilung der Euphausiaceen-Iarven aus den Planktonfängen der Deutsch-Britischen Antarktis-Expedition mit RRS "John Biscoe" im Februar 1982 dargestellt. Die Planktonfänge wurden mit dem RMT $1+8 \mathrm{~m}$ und dem Nansen-Schließ-Netz im Raum der antarktischen Halbinsel und der Scotia See durchgeführt. Vier Arten von Euphausiaceen-Larven kamen in diesem Gebiet vor. Euphausia superba war die dominante Art. 
EUPHAUSIID LARVAE IN PLANKTON SAMPLES FROM THE VICINITY OF THE ANTARCTIC PENINSUIA, FEBRUARY 1982

by Sigrid Marschall and Elke Mizdalski

1.

Introduction

The following data lists and distribution maps are the results of the identification work on euphausiid larvae caught during the Joint Biological Expedition on RRS "John Biscoe" in the austral summer of 1981/82. On the third leg of this expedition in February 1982 zooplankton was sampled at four time stations and on several profiles in five areas:

- Scotia Sea, including the Elephant Island region,

- Bransfield strait

- areas west of the Antarctic Peninsula

- North-western Weddell Sea

- South Georgia region.

For detailed information see HEMPEL \& HEYWOOD (1982), who give a description of cruise track, reports of individual working groups and preliminary results.

2 .

\section{Methods}

Two types of nets were used:

1. The RMT $1+8 \mathrm{~m}$ ("Rectangular Midwater Trawl") is a multiple opening and closing net system. For this report only material from the RMT 1 net was used. The RMT 1 net has a mouth area of approximately $1 \mathrm{~m}^{2}$ and a mesh-size of $320 \mu \mathrm{m} .76$ oblique hauls were performed with this net. Usually three depth strata were sampled: $200-140 \mathrm{~m}, 140 \mathrm{~m}$ - thermocline region (around $60 \mathrm{~m}$ ), thermocline region - below surface $(\sim 5 \mathrm{~m})$. These hauls were performed for studies on zooplankton and micronekton distribution and abundance.

2. The NCN (Nansen-Closing-Net) is a vertical net with a circular mouth opeñing of $70 \mathrm{~cm}$, a mesh-size of $200 \mu \mathrm{m}$ and a closing device which allows sampling discrete depth layers. It was used for 92 vertical hauls usually in depths layers from $2000-1000$, $1000-500 \mathrm{~m}, 500-200 \mathrm{~m}, 200-0 \mathrm{~m}$ to give more insight into the vertical distribution of krill eggs and larvae during the developmental ascent.

The samples were fixed in $4 \%$ chalk-buffered formaldehyde solution.

For station 1 ists and comments to stations, see PIATKOWSKI (1983).

Figures 1-4 give the locations of stations, at which zooplanktonsampling was carried out.

The early life history stages of Euphausiids from the RMT-1-samples were sorted into the following categories:

- Euphausia superba: eggs, naupliar stages, calyptopes and furciliae.

- Euphausiid larvae other than krill: naupliar stages, calyptopes and furciliae. 


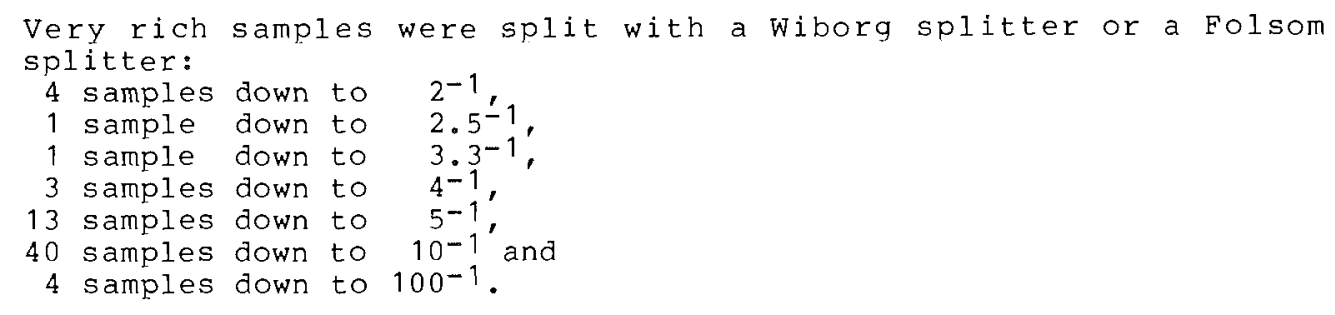

The early life history stages of Euphausidis from the NCN-samples were sorted as described for the RMT 1-samples.

A Wiborg splitter was used to obtain subsamples. 7 samples were split down to $10^{-1}$, 1 sample down to $100^{-1}$.

The following data lists give the results of the identification of euphausidd larvae:

- the first list contains data on Euphausia superba eags and larvae,

- the second list gives data on euphausidd larvae other than krill, i.e. Thysanoessa sp., Euphausia frigida and Euphausia crystallorophias. The larvae of the qenus Thysanoessa (T. vicina and T. macrural are given as Thysanoessa sp., because they can not be distinguished.

Number of individuals is given as $\mathrm{n} / 1000 \mathrm{~m}^{3}$.

3. Results

Euphausia superba larvae were found in the entire investigated area with numbers up to $20000 / 1000 \mathrm{~m}^{3}$. The main areas of distribution were around Elephant Island, the Bransfield strait and the Antarctic sound (Figs. 5-6).

of the other euphausid larvae, Thysanoessa sp. was the dominant species with numbers up to more than $3000 / 1000 \mathrm{~m}^{3}$. It was found in almost every sample but at higher concentrations around south Georgia and the area west of the South Shetland Islands (Fiq. 7a). In the Bransfield strait Thysanoessa $\mathrm{sp.} \mathrm{had} \mathrm{a} \mathrm{homogeneous} \mathrm{distribu-}$ tion (Fiq. 7b).

E. crystallorophias and E. frigida were only found in a few hauls. The main area of distribution of E. friaida larvae was around South Georgia and in the scotia sea (up to 231/1000 $\mathrm{m}^{3}$, Figs. 8-9). Few larvae were caught along the south shetland Islands.

These results are in accordance with those of HEMPEL \& MARSCHOFF $(1980)$.

Large numbers of E. crystallorophias occurred in the laqoon of Deception Island $\left(12 \frac{114 / 1000 \mathrm{~m}^{3}, \mathrm{Fiq} .}{1 \mathrm{~b})}\right.$, where it was the only euphausiid species.

An interesting fact was the occurrence of larvae of all 4 euphausid species in the Antarctic Sound. 


\section{Acknowledgement}

We thank all sorters for their useful help. G. Dieckmann kindly revised the English text.

4.

References

HEMPEL, G. \& R.B. HEYWOOD (1982): Joint Biological Expedition on RRS John Biscoe, February 1982. Ber. Polarforsch. $\underline{5}, 39 \mathrm{pp}$.

HEMPEL, I. \& E. MARSCHOFF (1980): Euphausiid larvae in the Atlantic Sector of the Southern Ocean. Meeresforsch. 28, 32-47.

PIATKOWSKI, U. (1983): Joint Biological Expedition on RRS "John Biscoe", February 1982 (II). Data of micronekton and zooplankton hauls. Ber. Polarforsch. 11, $40 \mathrm{pp}$. 


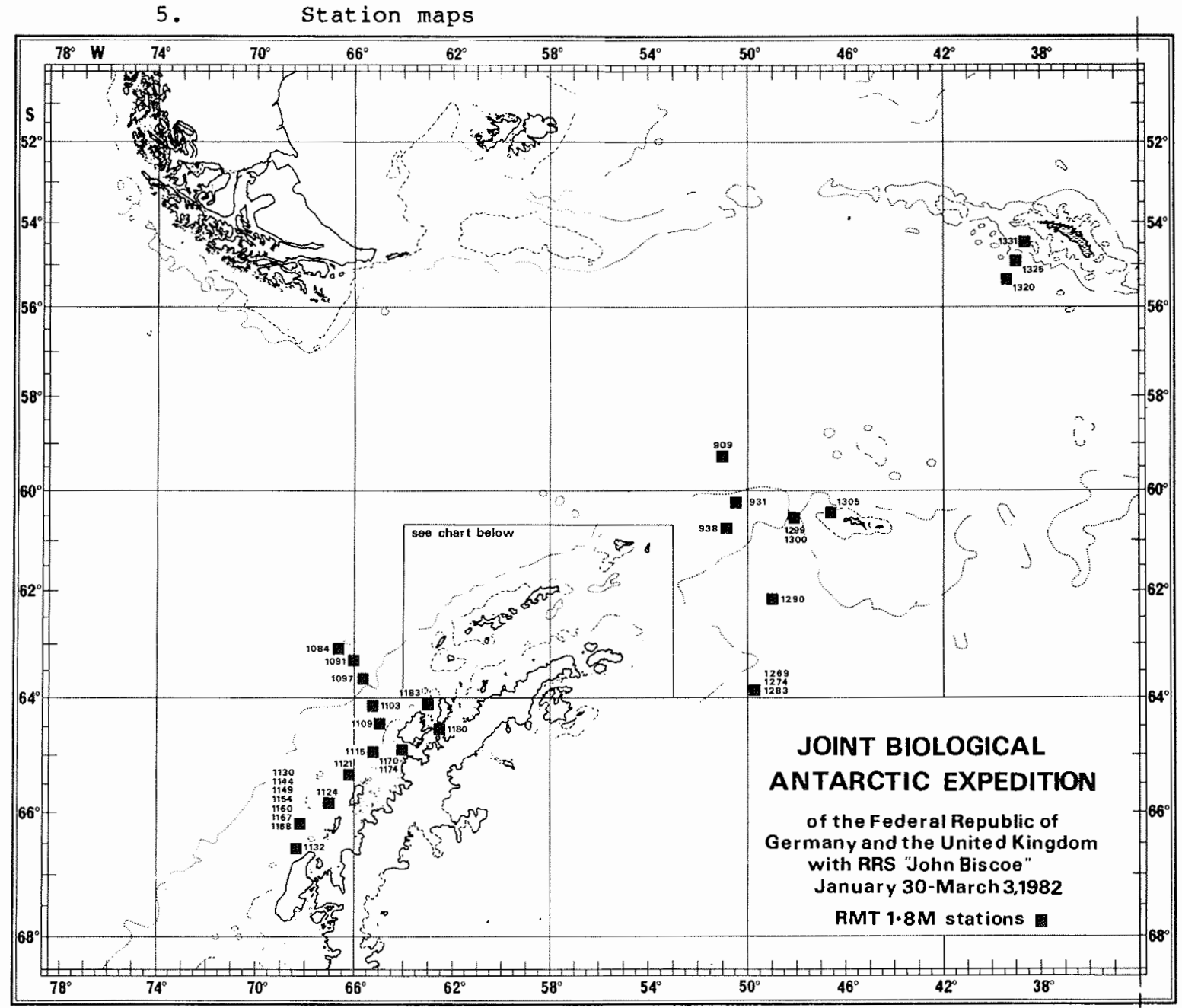

Fig. 1 RMT $1+8 \mathrm{~m}$ stations of whole investigated area. (From PIATKOWSKI, 1983). 


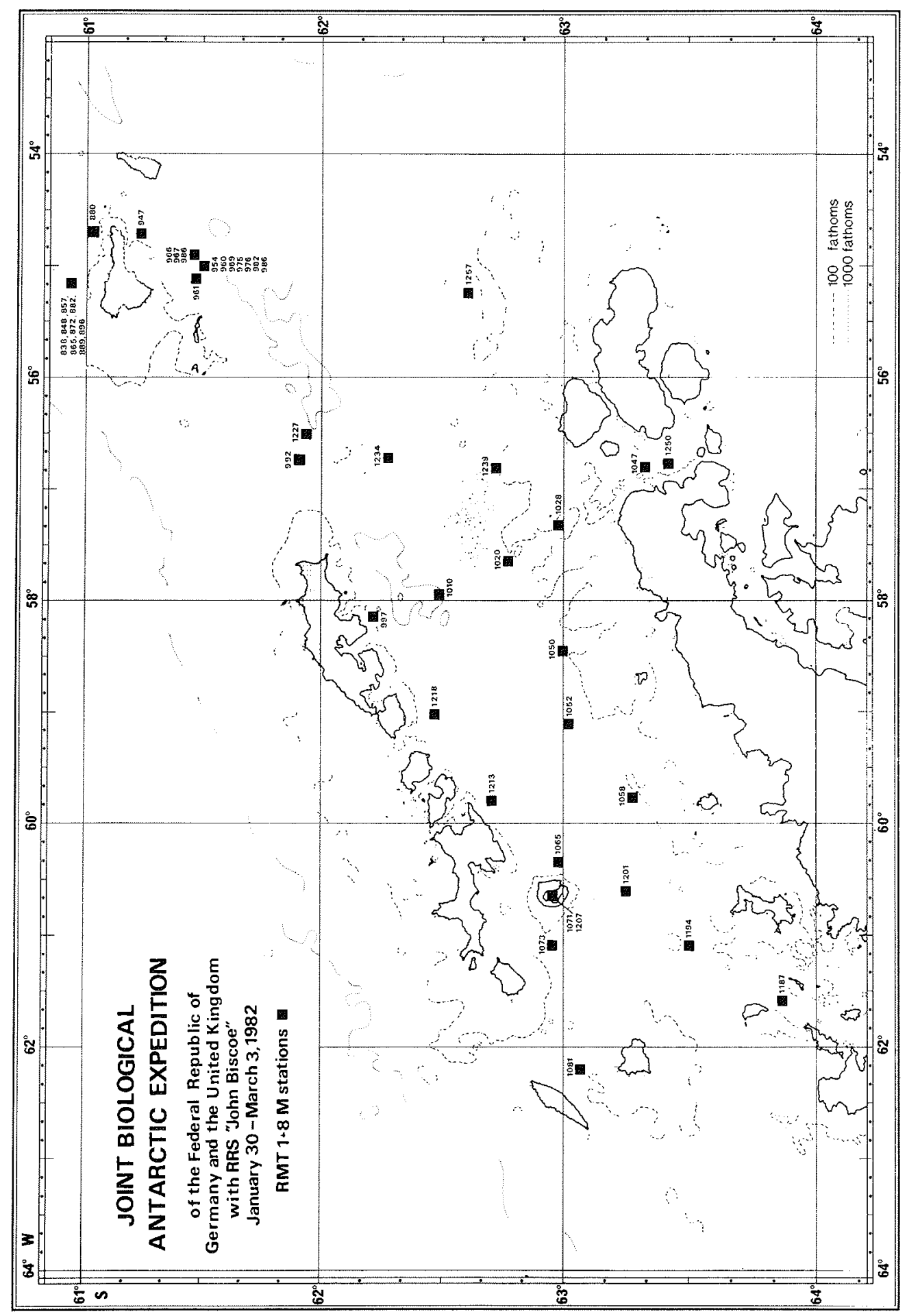

Fig. 2 RMT $1+8 \mathrm{~m}$ stations in Bransfield strait and adjacent waters. (From PIATKOWSKI, 1983). 


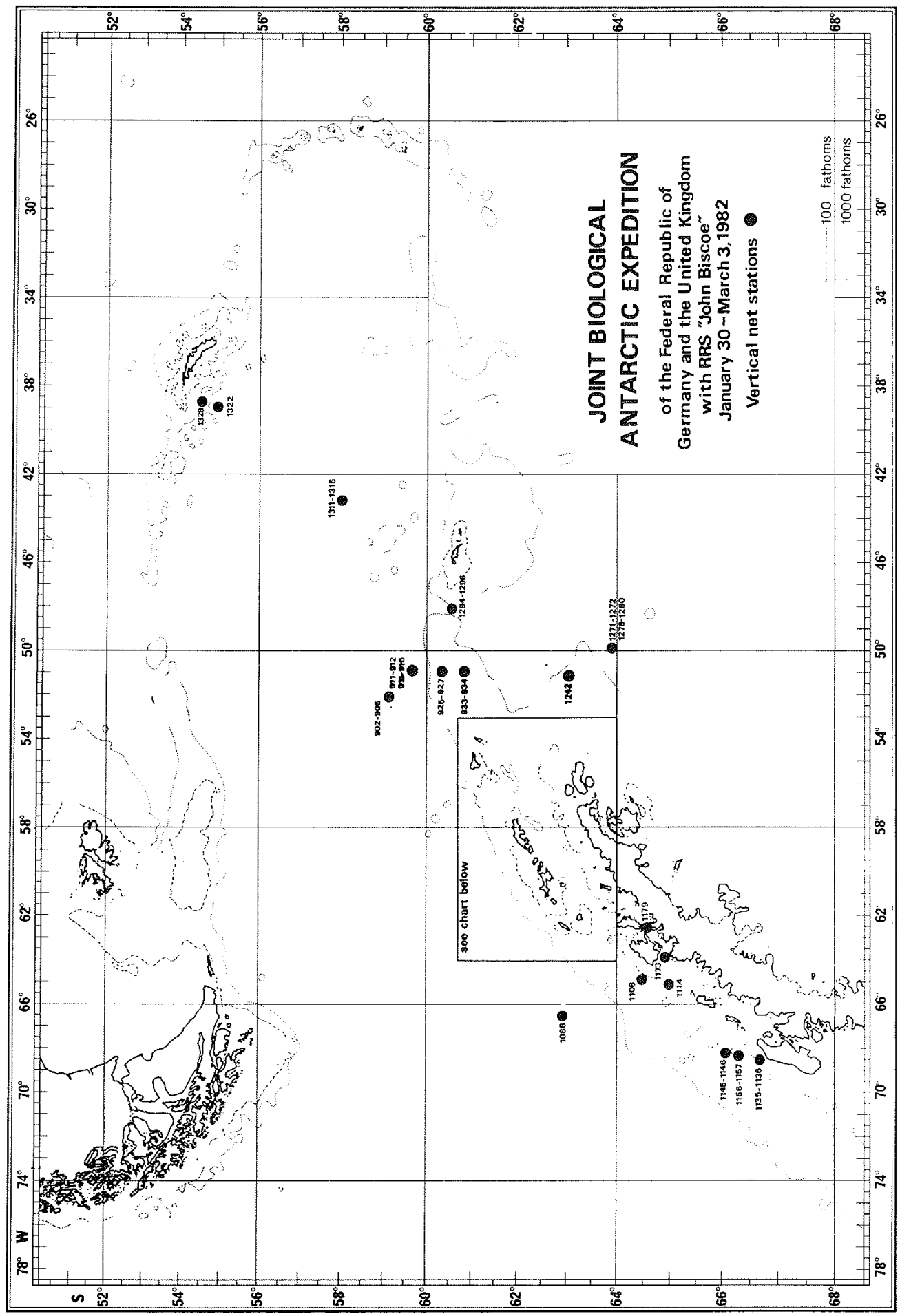

Fiq. 3 Vertical net stations of whole investigated area. (From PIATKOWSKI, 1983). 


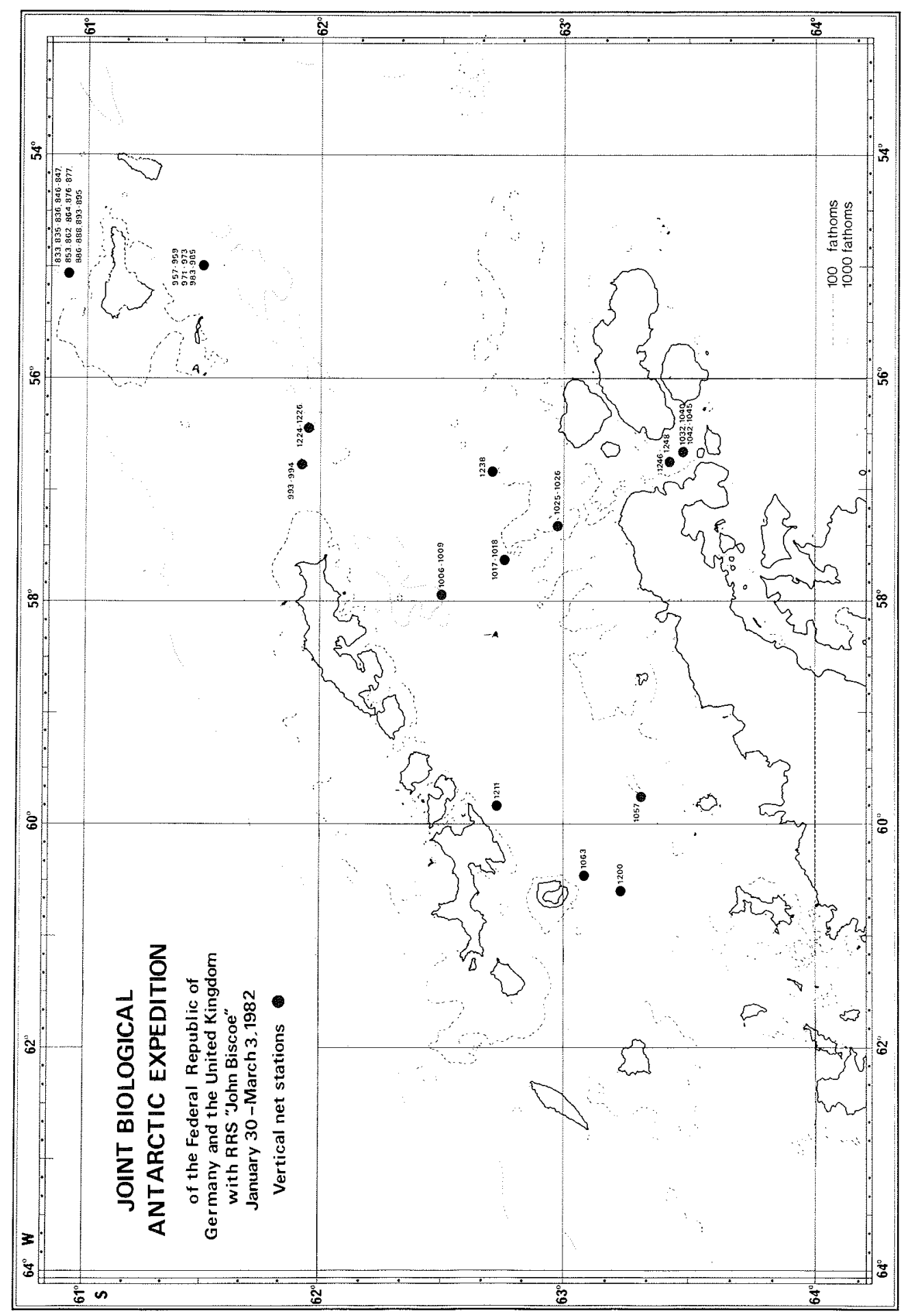

Fia. 4 Vertical net stations in Bransfield strait and adjacent waters.

(From PIATKOWSKI, 1983). 
6.

\section{Distribution maps}

The following maps include only those stations where the entire water column was sampled.

The NCN-samples did not yield quantitative data on furciliae. These stages are therefore not included in the maps. 

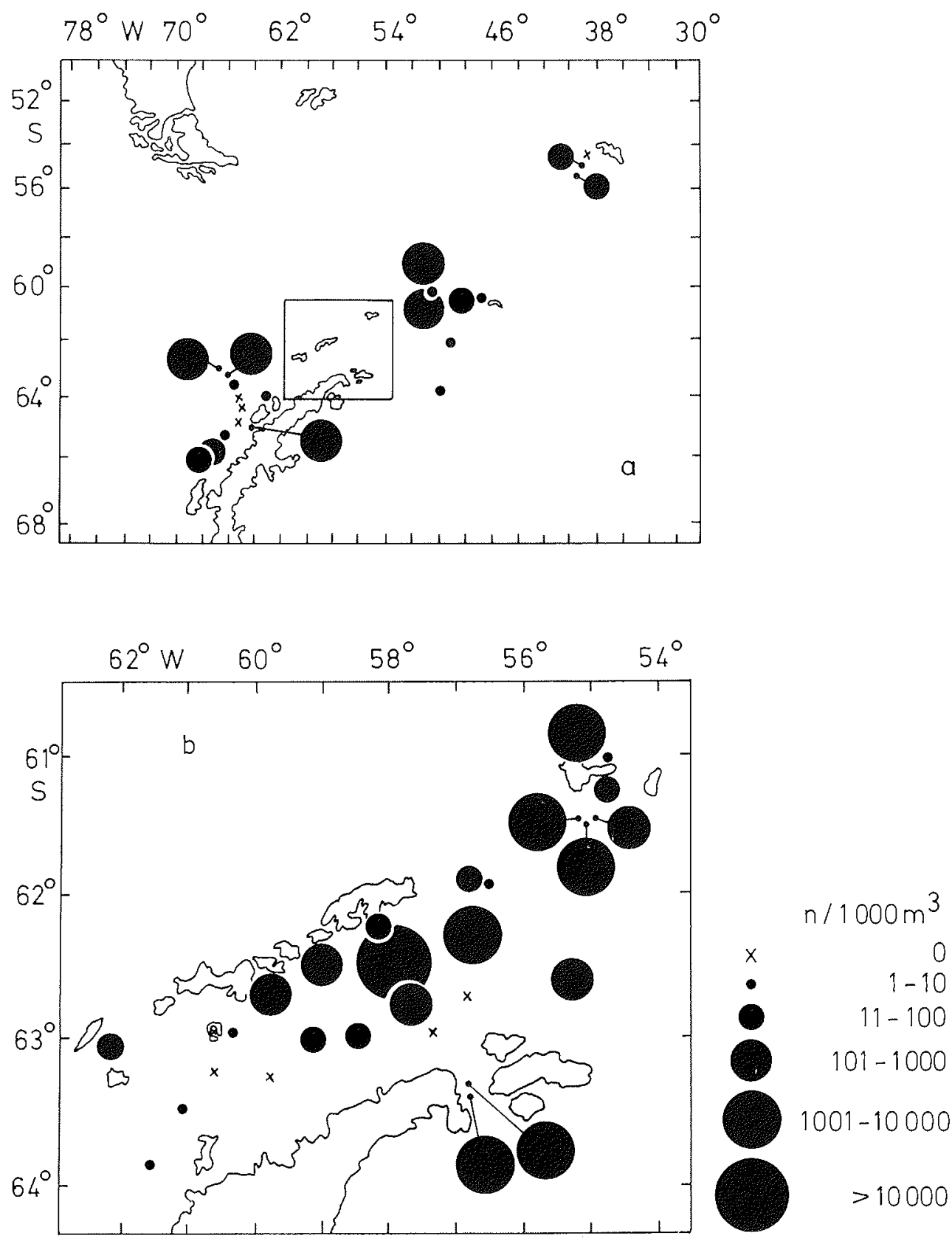

Fig. 5 Horizontal distribution of Euphausia superba larvae, RMT 1, $320 \mu \mathrm{m}$. 

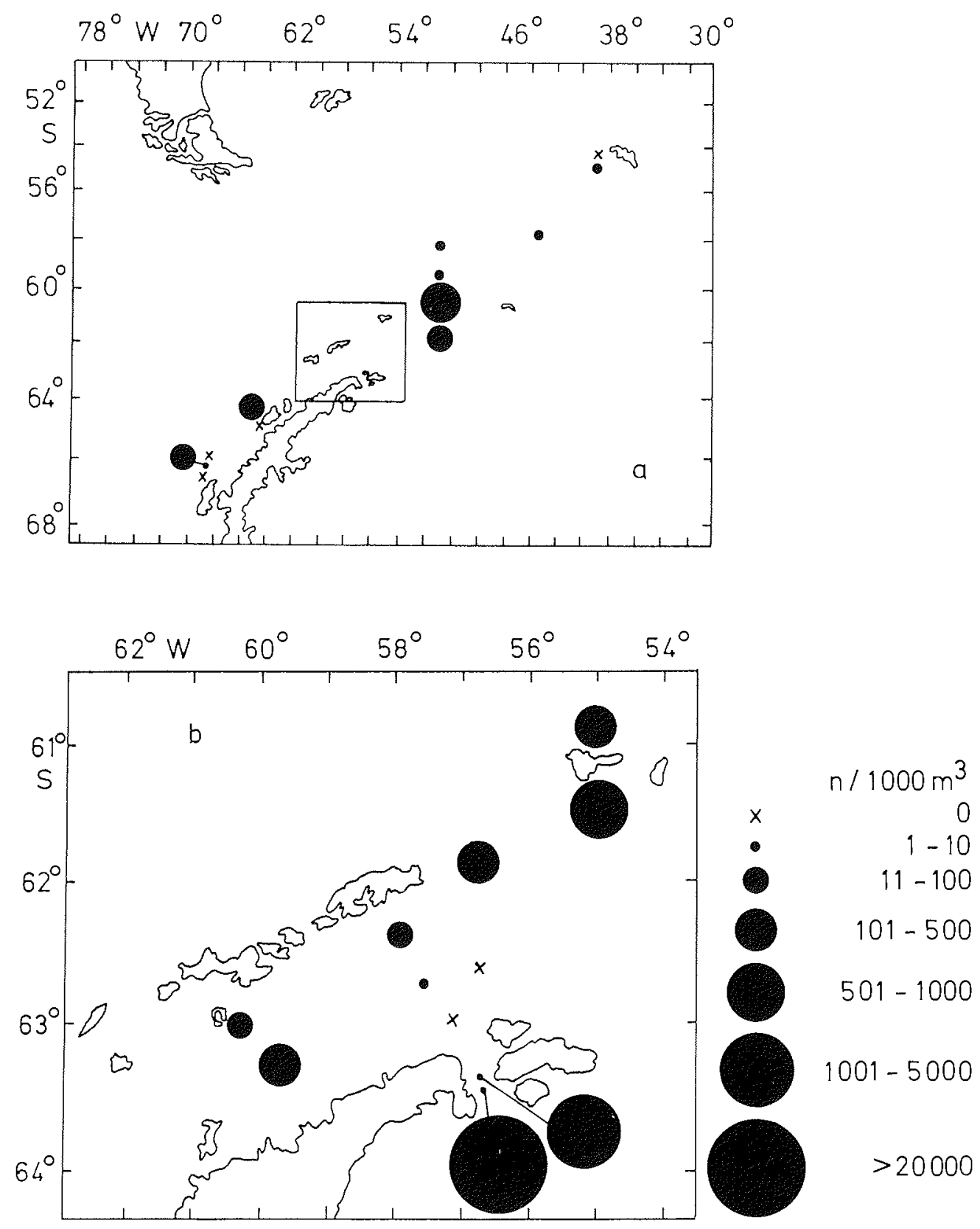

Fig. 6 Horizontal distribution of Euphausia superba larvae except furciliae, NCN, $200 \mu \mathrm{m}$. 

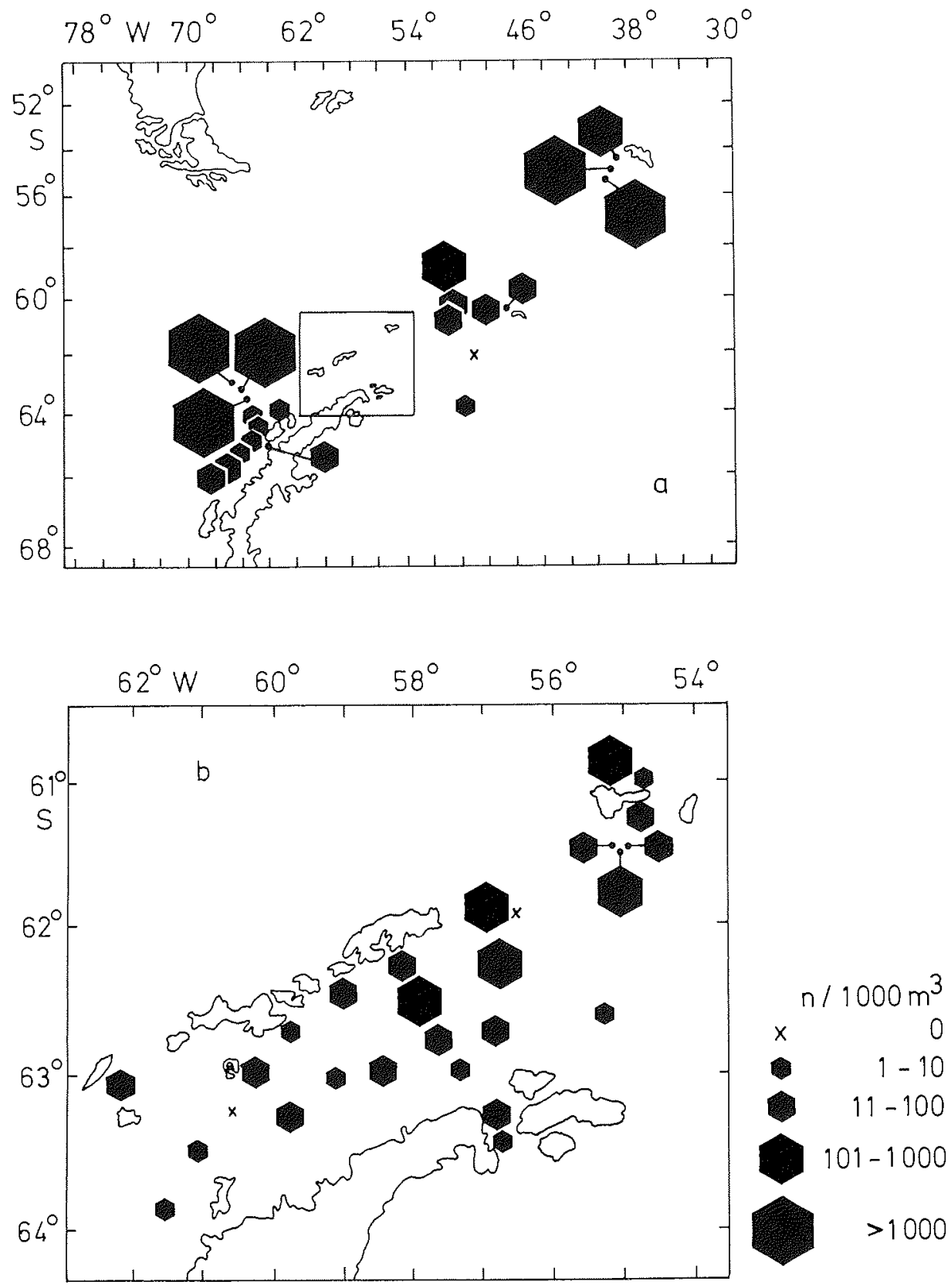

Fig. 7 Horizontal distribution of Thysanoessa sp. larvae, RMT 1 , $320 \mu \mathrm{m}$. 

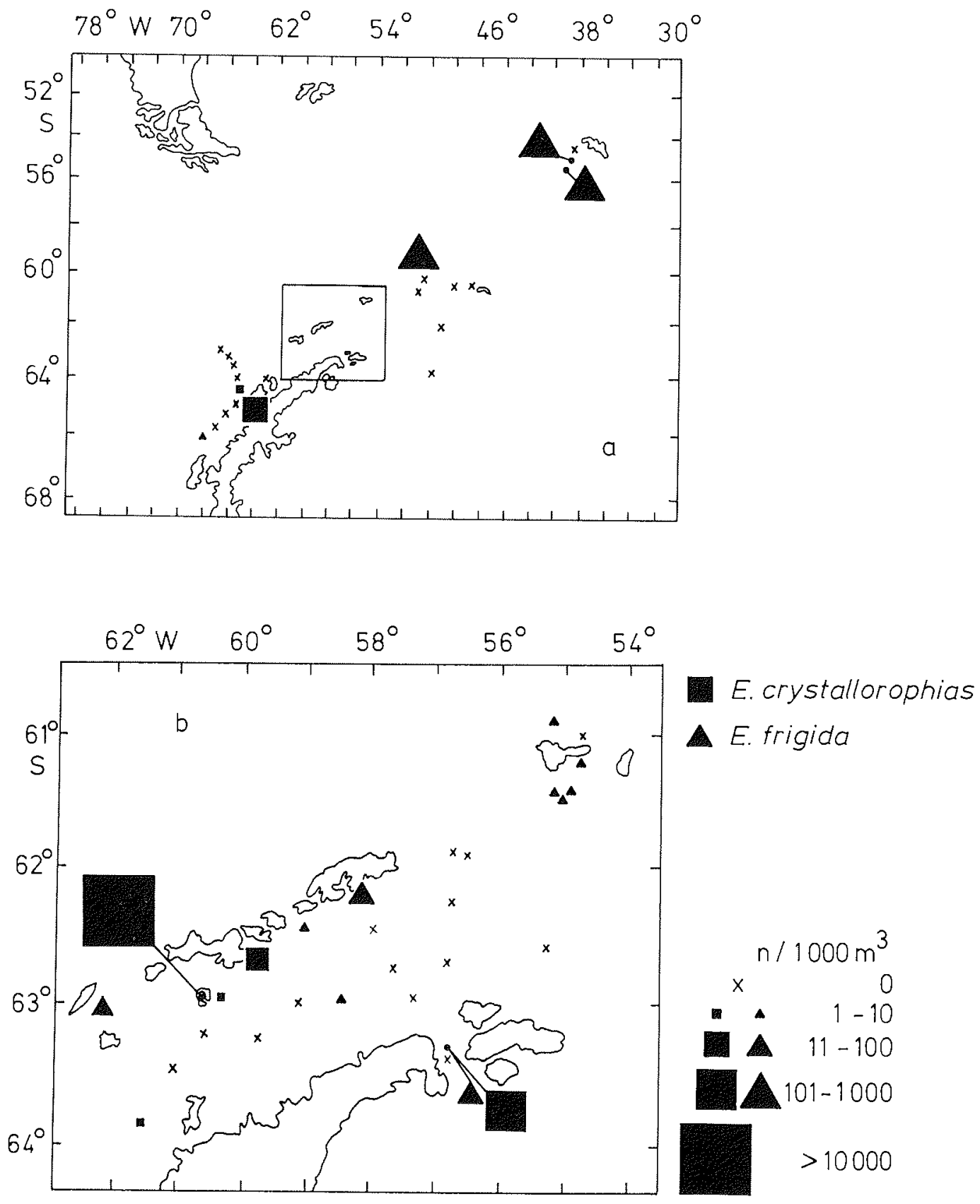

Fig. 8 Horizontal distribution of Euphausia crystallorophias and Euphausia frigida larvae, RMT $\frac{1}{1}, 320 \mu \mathrm{m}$. 

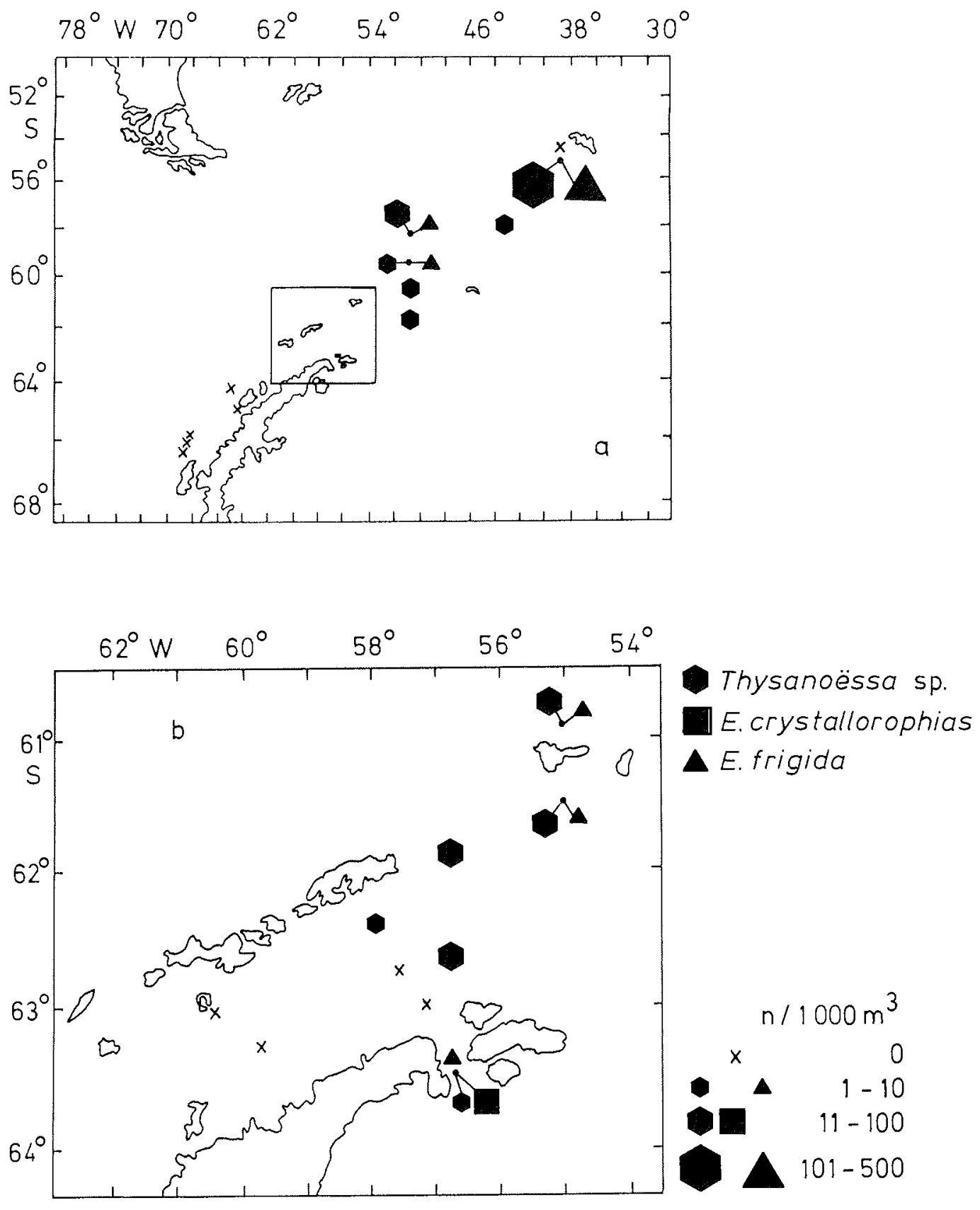

Fig. 9 Horizontal distribution of Thysanoessa sp., Euphausia crystallorophias and Euphausia Erigida larvae except furciliae, NCN, $200 \mu \mathrm{m}$. 
$\vec{\infty} \quad$ Antarctic Expedition 1981/82 RRS "John Biscoe". Distribution of Euphausia superba eggs and larvae.

\begin{tabular}{|c|c|c|c|c|c|c|c|c|c|c|c|c|c|c|}
\hline \multirow{2}{*}{ Stat./Haul } & $\begin{array}{l}\text { Date } \\
1982\end{array}$ & Gear & $\begin{array}{l}\text { Haul } \\
\text { depth } \\
\text { (m) }\end{array}$ & Eggs & $\begin{array}{l}\text { Nau- } \\
\text { plii }\end{array}$ & $\begin{array}{l}\text { Meta- } \\
\text { naupl. }\end{array}$ & I & II & III & Early & an & Late & $\begin{array}{l}\text { Total } \\
\text { number of } \\
\text { larvae }\end{array}$ & Comment \\
\hline & Febx & & & & & & & & & & & & & \\
\hline $835 / 2$ & 2 & $\mathrm{NCN}$ & $1000-200$ & 20 & 7 & 20 & 68 & 0 & 0 & 0 & 0 & 0 & 94 & - \\
\hline $836 / 3$ & 2 & $\mathrm{NCN}$ & $200-\quad 0$ & 39 & 0 & 0 & 104 & 0 & 0 & 0 & 0 & 0 & 104 & - \\
\hline $846 / 4$ & 2 & $\mathrm{NCN}$ & 500 & 15 & 0 & 0 & 292 & 0 & 0 & 0 & 0 & 0 & 292 & - \\
\hline $847 / 5$ & 2 & $\mathrm{NCN}$ & $1000-500$ & 0 & 16 & 37 & 73 & 0 & 0 & 0 & 0 & 0 & 125 & - \\
\hline $853 / 6$ & 2 & $\mathrm{NCN}$ & $2000-1000$ & 5 & 221 & 148 & 5 & 0 & 0 & 0 & 0 & 0 & 374 & - \\
\hline \multirow[t]{2}{*}{$848 / 2$} & 2 & RMT 1-1 & $200-160$ & 18 & 0 & 0 & 6 & 0 & 0 & 0 & 0 & 0 & 6 & - \\
\hline & & RMT 1-2 & $160-125$ & 52 & 0 & 6 & 10 & 0 & 0 & 0 & 0 & 0 & 16 & - \\
\hline \multirow{3}{*}{$857 / 3$} & 2 & RMT1-1 & $255-135$ & 32 & 0 & 0 & 520 & 0 & 0 & 3 & 0 & 0 & 523 & - \\
\hline & & RMPT 1-2 & $135-70$ & 4 & 0 & 0 & 0 & 0 & 0 & 0 & 0 & 0 & 0 & - \\
\hline & & RMT 1-3 & $70-\quad 20$ & 0 & 0 & 0 & $19 *$ & 0 & 0 & 0 & 0 & 0 & 19 & $\begin{array}{l}\text { * partly } \\
\text { fraqments }\end{array}$ \\
\hline $862 / 7$ & 2 & $\mathrm{NCN}$ & $2019-1000$ & 34 & 237 & 482 & 13 & 0 & 0 & 0 & 0 & 0 & 732 & - \\
\hline $863 / 8$ & 3 & $\mathrm{NCN}$ & $1000-500$ & 15 & 57 & 188 & 125 & 0 & 0 & 0 & 0 & 0 & 370 & - \\
\hline $864 / 9$ & 3 & $\mathrm{NCN}$ & $500-200$ & 9 & 78 & 122 & 70 & 0 & 0 & 0 & 0 & 0 & 270 & - \\
\hline \multirow[t]{3}{*}{$865 / 4$} & 3 & RMT1-1 & $200-140$ & 10 & 0 & 0 & 0 & 0 & 0 & 0 & 0 & 0 & 0 & - \\
\hline & & RMT1-2 & $140-60$ & 0 & 0 & 0 & 0 & 0 & 0 & 0 & 0 & 0 & 0 & - \\
\hline & & RMT 1-3 & $60-\quad 10$ & 0 & 0 & 0 & 0 & 0 & 0 & 0 & 0 & 0 & 0 & - \\
\hline \multirow[t]{3}{*}{$872 / 5$} & 3 & RMT1-1 & $200-145$ & 59 & 0 & 0 & 12 & 0 & 0 & 0 & 0 & 0 & 12 & - \\
\hline & & RMT1-2 & $145-70$ & 139 & 0 & 0 & 94 & 0 & 0 & 0 & 0 & 0 & 94 & - \\
\hline & & RMT 1-3 & $70-15$ & 0 & 0 & 0 & 7 & 0 & 0 & 0 & 0 & 0 & 7 & - \\
\hline
\end{tabular}




\begin{tabular}{|c|c|c|c|c|c|c|c|c|c|c|c|c|c|c|}
\hline \multirow{2}{*}{ Stat./Haul } & \multirow{2}{*}{$\begin{array}{l}\text { Date } \\
1982 \\
\text { Febr }\end{array}$} & \multirow[t]{2}{*}{ Gear } & \multirow[t]{2}{*}{$\begin{array}{l}\text { Haul } \\
\text { depth } \\
\text { (m) }\end{array}$} & \multirow[t]{2}{*}{ Eggs } & \multirow[t]{2}{*}{$\begin{array}{l}\text { Nau- } \\
\text { plii }\end{array}$} & \multirow[t]{2}{*}{$\begin{array}{l}\text { Meta- } \\
\text { naupl. }\end{array}$} & \multirow{2}{*}{\multicolumn{2}{|c|}{$\begin{array}{l}\text { Calyptopis } \\
\text { II }\end{array}$}} & \multirow[t]{2}{*}{ III } & \multicolumn{3}{|c|}{$\begin{array}{l}\text { Furcilia } \\
\text { Early Mean Late }\end{array}$} & $\begin{array}{l}\text { Total } \\
\text { number of } \\
\text { larvae }\end{array}$ & \multirow[t]{2}{*}{ Comment } \\
\hline & & & & & & & & & & & & & & \\
\hline $876 / 10$ & 3 & $\mathrm{NCN}$ & $2000-1000$ & 8 & 42 & 86 & 0 & 0 & 0 & 0 & 0 & 0 & 127 & - \\
\hline $877 / 11$ & 3 & $\mathrm{NCN}$ & $1000-500$ & 26 & 52 & 52 & 10 & 0 & 0 & 0 & 0 & 0 & 115 & - \\
\hline $880 / 6$ & 3 & RMT1-1 & $200-140$ & 29 & 0 & 0 & 0 & 0 & 0 & 3 & 0 & 0 & 3 & - \\
\hline & & RMT1-2 & $140-70$ & 27 & 0 & 0 & 0 & 0 & 0 & 0 & 0 & 0 & 0 & - \\
\hline & & RMT 1-3 & $70-\quad 5$ & 15 & 0 & 0 & 0 & 0 & 0 & 0 & 0 & 0 & 0 & - \\
\hline $882 / 7$ & 3 & RMT1-1 & $200-140$ & 118 & 0 & 0 & 42 & 1 & 0 & 0 & 0 & 0 & 44 & - \\
\hline & & RMT1-2 & $140-70$ & 57 & 0 & 0 & 13 & 0 & 0 & 0 & 0 & 0 & 13 & - \\
\hline & & RMT1-3 & $70-\quad 10$ & 10 & 0 & 0 & 0 & 0 & 0 & 0 & 0 & 0 & 0 & - \\
\hline $886 / 12$ & 4 & $\mathrm{NCN}$ & $2000-1000$ & 24 & 210 & 429 & 0 & 3 & 0 & 0 & 0 & 0 & 642 & - \\
\hline $887 / 13$ & 4 & $\mathrm{NCN}$ & $1000-500$ & 5 & 42 & 224 & 47 & 0 & 0 & 0 & 0 & 0 & 313 & - \\
\hline $888 / 14$ & 4 & $\mathrm{NCN}$ & $500-\quad 0$ & 0 & 0 & 5 & 630 & 5 & 0 & 0 & 0 & 0 & 641 & - \\
\hline $889 / 8$ & 4 & RMT1-1 & $200-100$ & 73 & 0 & 0 & 2140 & 38 & 0 & 0 & 0 & 0 & 2177 & - \\
\hline & & RVTT1-2 & $100-85$ & 133 & 0 & 0 & 8447 & 192 & 0 & 89 & 89 & 0 & 8817 & - \\
\hline & & RMT 1-3 & $85-\quad 20$ & 0 & 0 & 0 & 7205 & 432 & 0 & 0 & 0 & 0 & 7637 & - \\
\hline $893 / 15$ & 4 & $\mathrm{NCN}$ & $500-\quad 0$ & 78 & 5 & 10 & 953 & 42 & 0 & 0 & 0 & 0 & 1010 & - \\
\hline $894 / 16$ & 4 & $\mathrm{NCN}$ & $1000-500$ & 25 & 10 & 412 & 328 & 0 & 0 & 0 & 0 & 0 & 750 & - \\
\hline $895 / 17$ & 4 & $\mathrm{NCN}$ & $2350-1000$ & 6 & 21 & 96 & 8 & 0 & 0 & 0 & 0 & 0 & 125 & - \\
\hline $896 / 9$ & 4 & RMT1-1 & $200-140$ & 61 & 0 & 0 & 23 & 4 & 0 & 0 & 0 & 0 & 27 & - \\
\hline & & RMT 1-2 & $140-\quad 65$ & 24 & 0 & 0 & 46 & 0 & 0 & 0 & 0 & 0 & 46 & - \\
\hline & & RMT1-3 & $65-\quad 15$ & 0 & 0 & 0 & 5 & 0 & 0 & 0 & 0 & 0 & 5 & - \\
\hline $902 / 18$ & 5 & $\mathrm{NCN}$ & $2000-1000$ & 0 & 0 & 8 & 0 & 0 & 0 & 0 & 0 & 0 & 8 & - \\
\hline $903 / 19$ & 5 & $\mathrm{NCN}$ & $1000-500$ & 0 & 0 & 5 & 0 & 0 & 0 & 0 & 0 & 0 & 5 & - \\
\hline $904 / 20$ & 5 & NCN & $500-200$ & 0 & 0 & 0 & 35 & 0 & 0 & 0 & 0 & 0 & 35 & - \\
\hline $905 / 21$ & 5 & $\mathrm{NCN}$ & $200-\quad 0$ & 0 & 0 & 0 & 0 & 0 & 0 & 0 & 0 & 0 & 0 & - \\
\hline
\end{tabular}




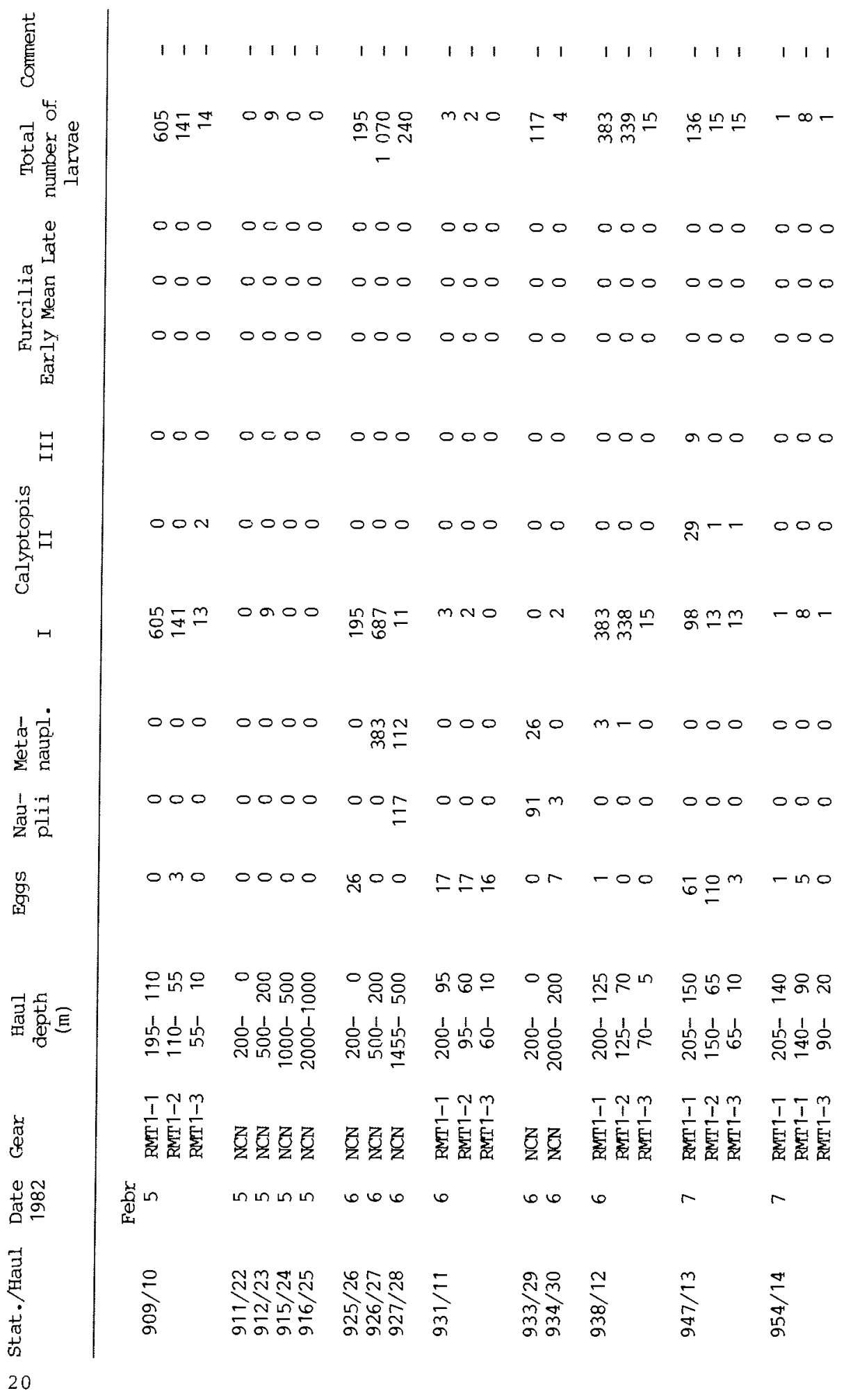




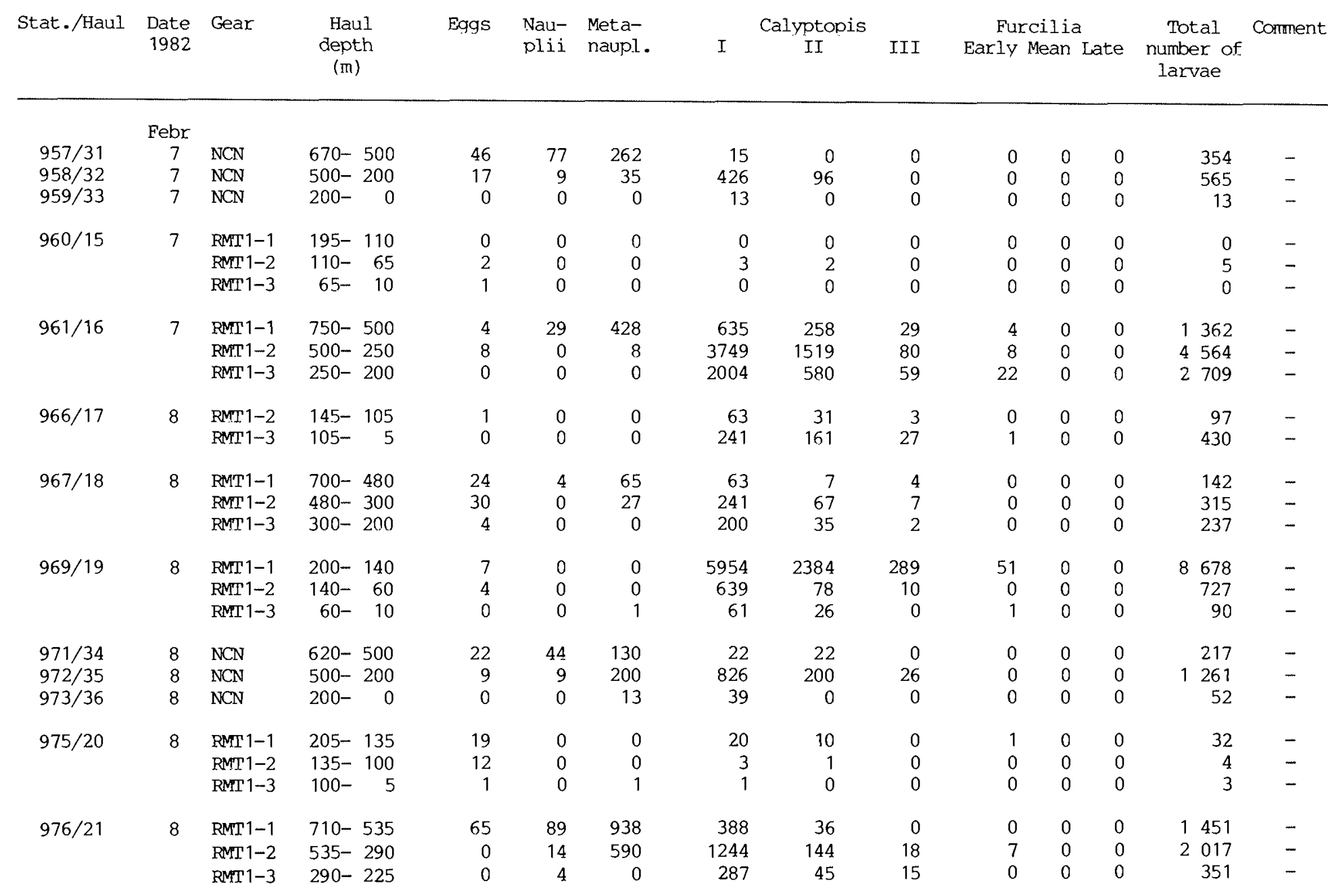




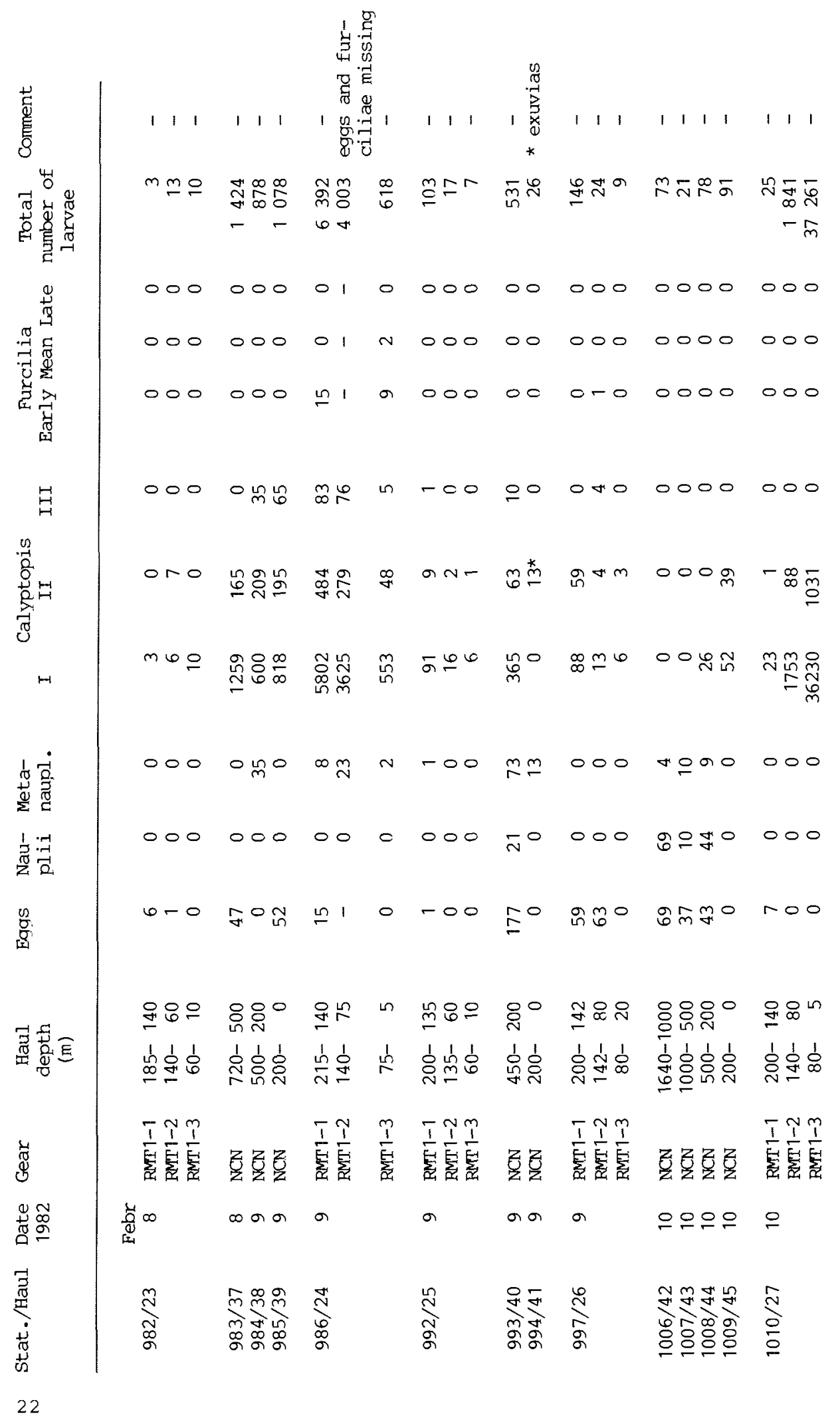




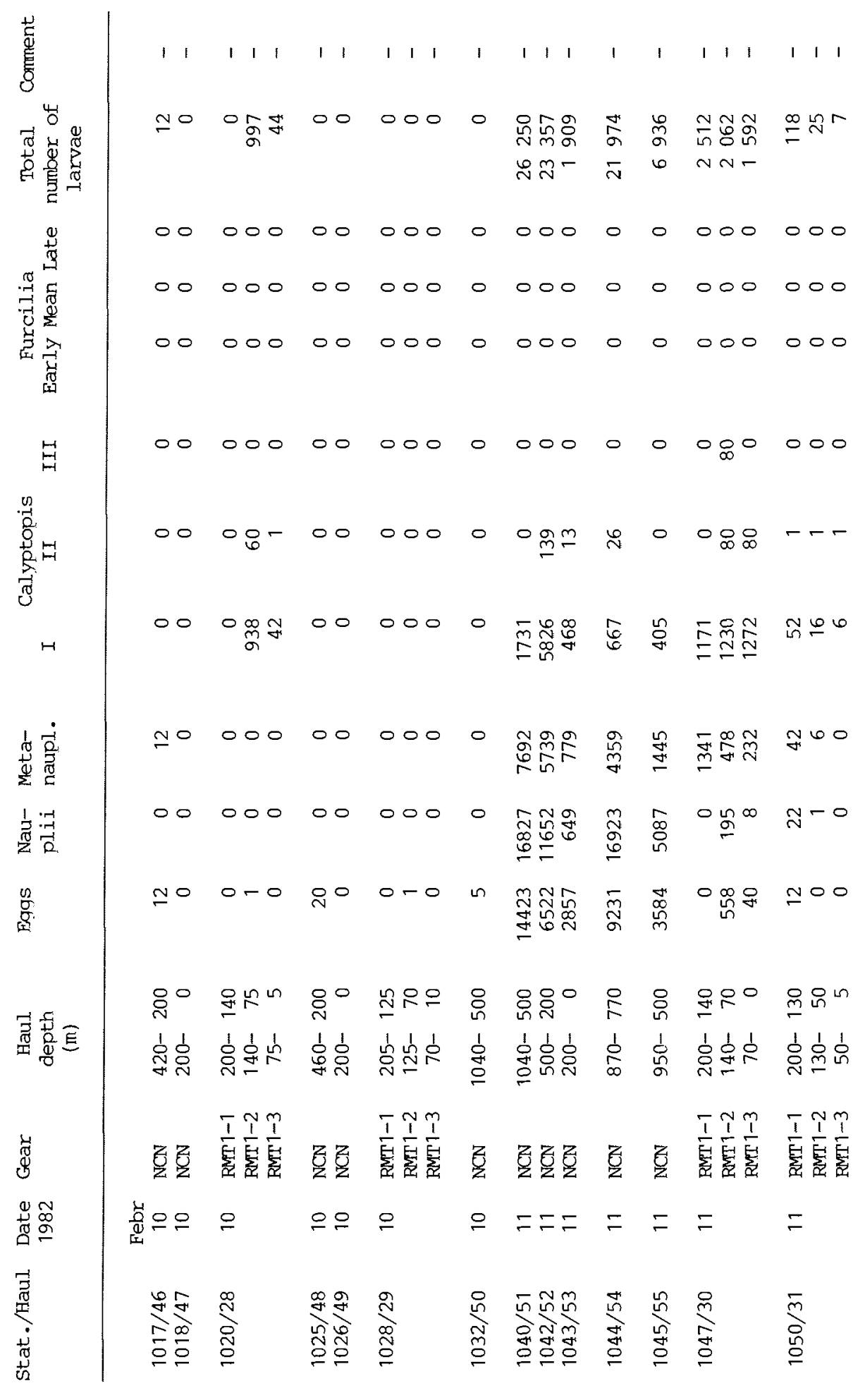




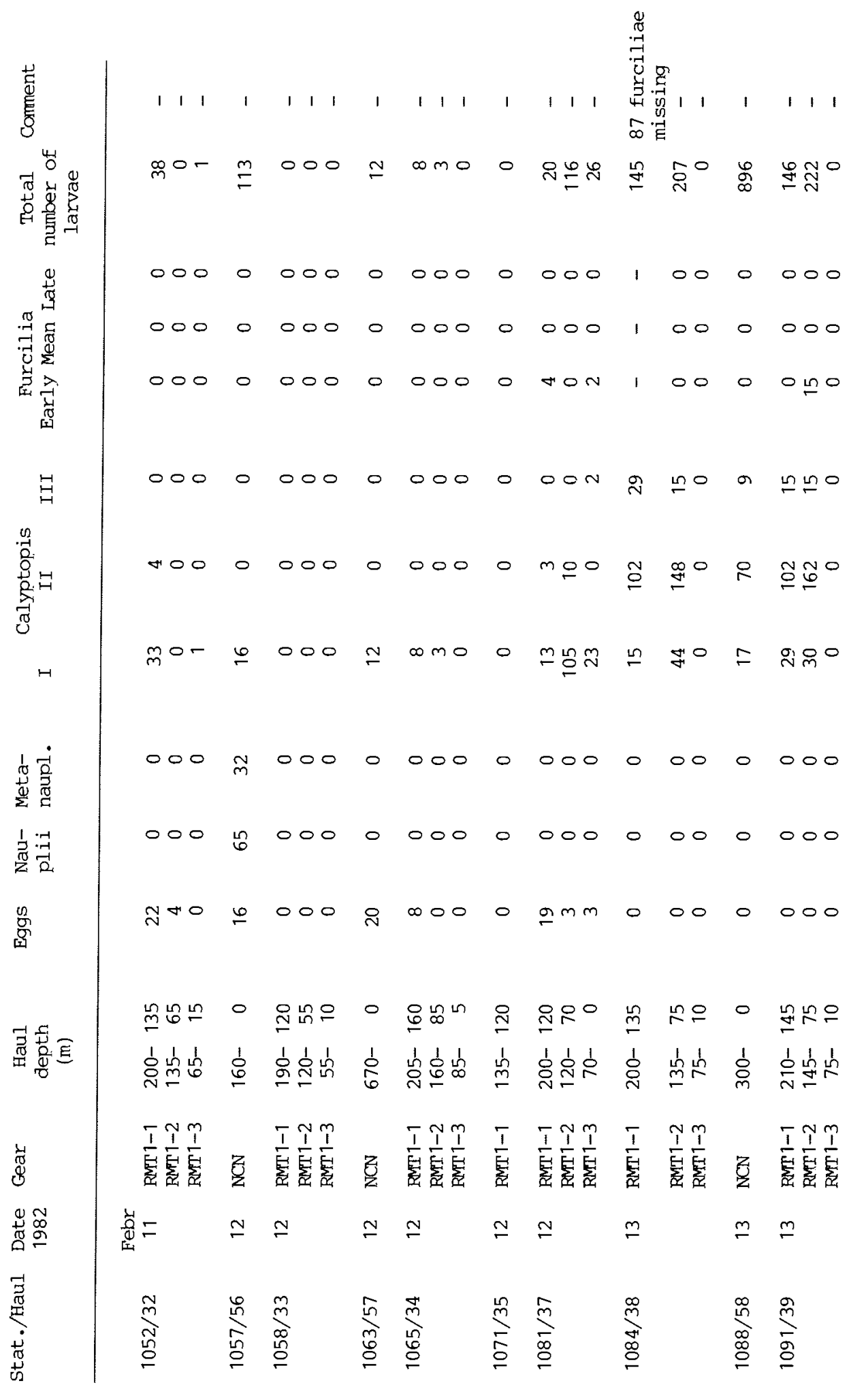




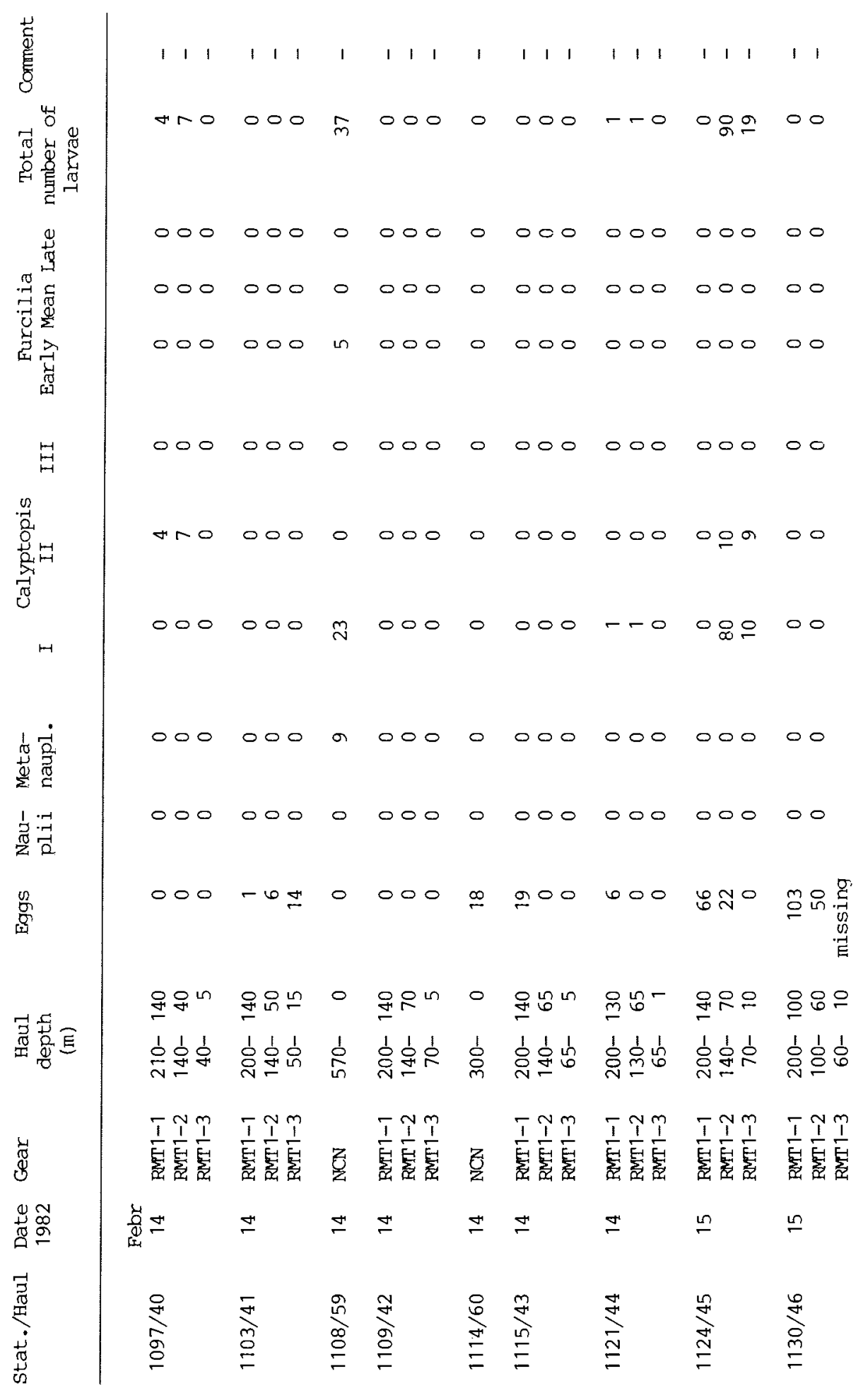




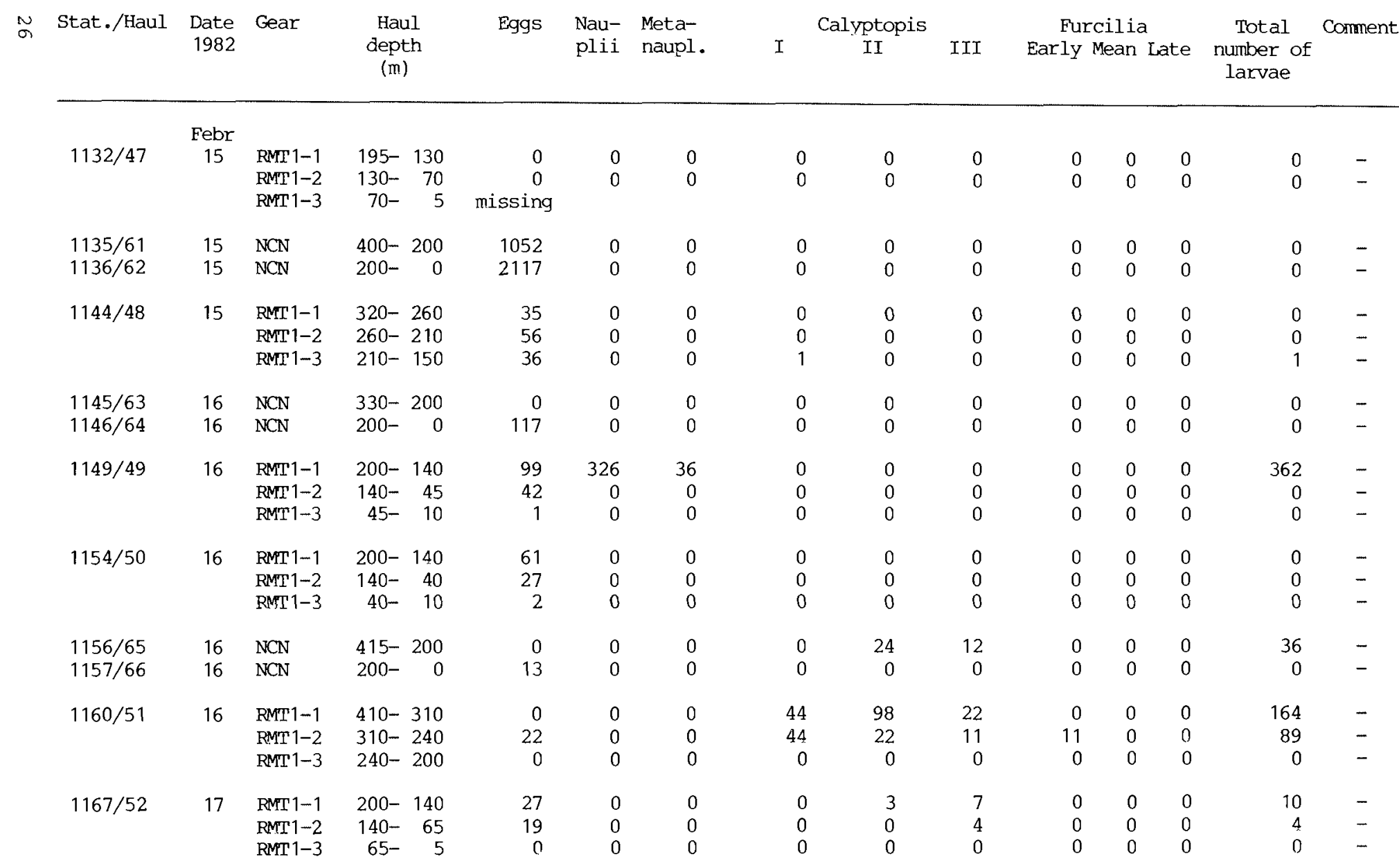




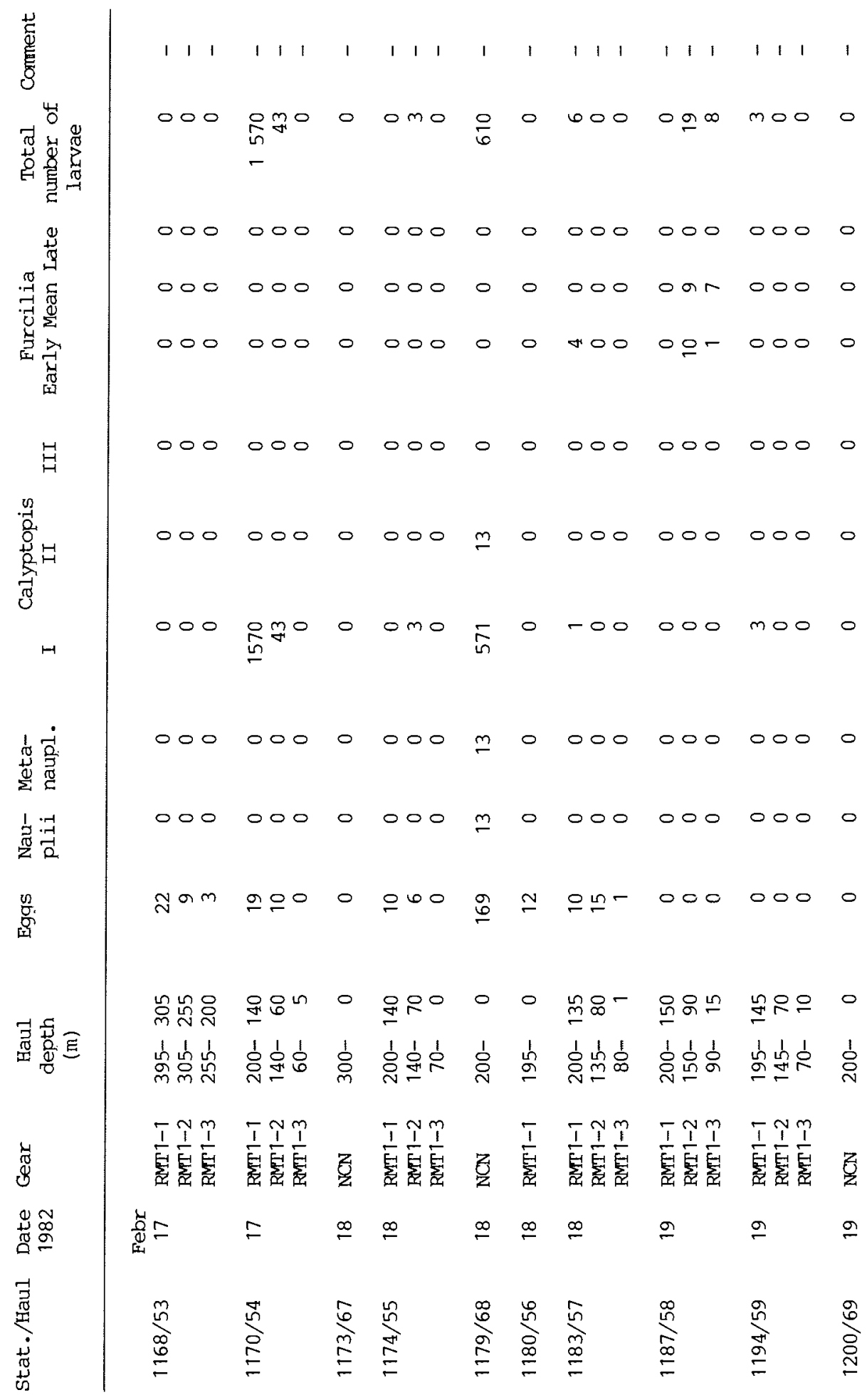




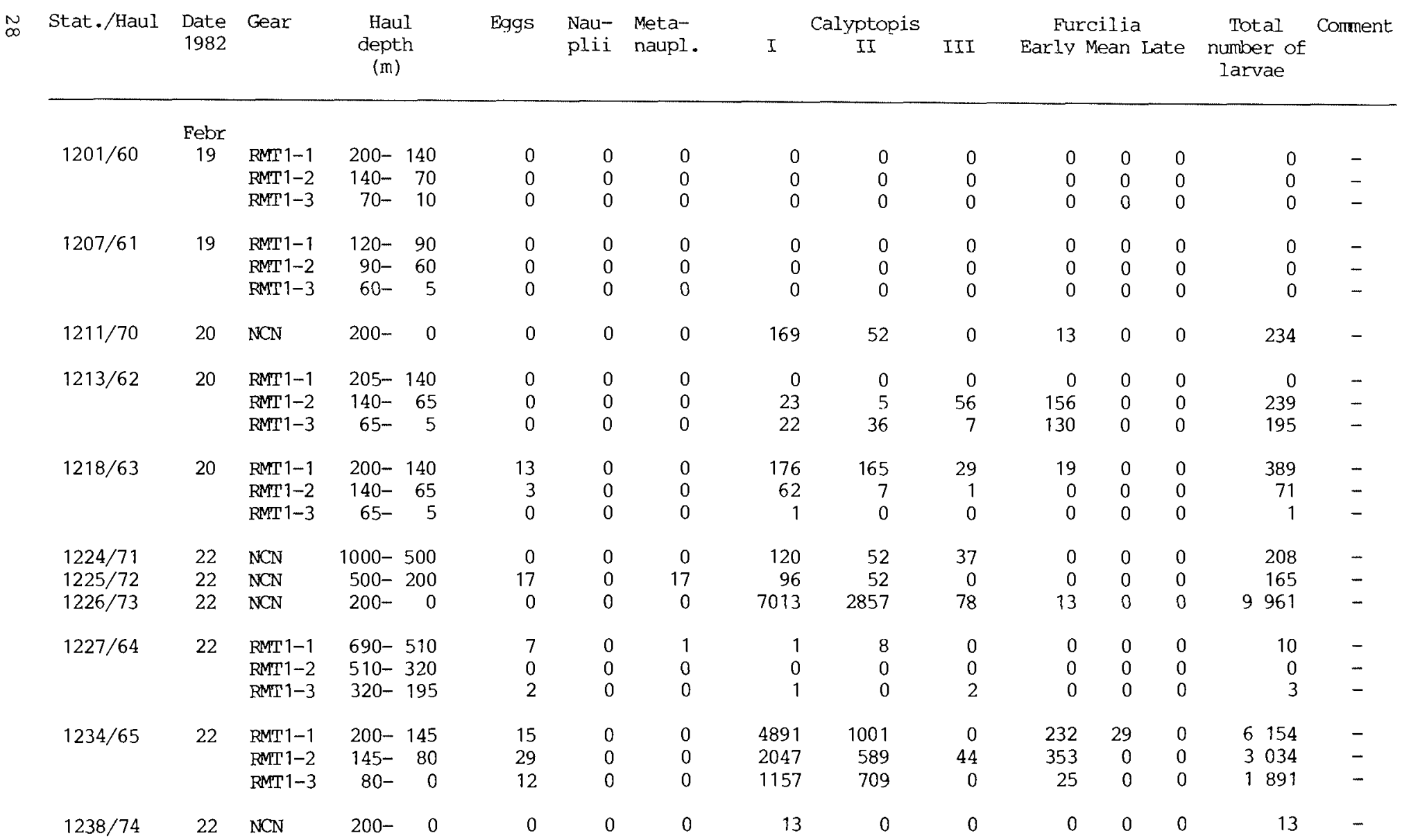




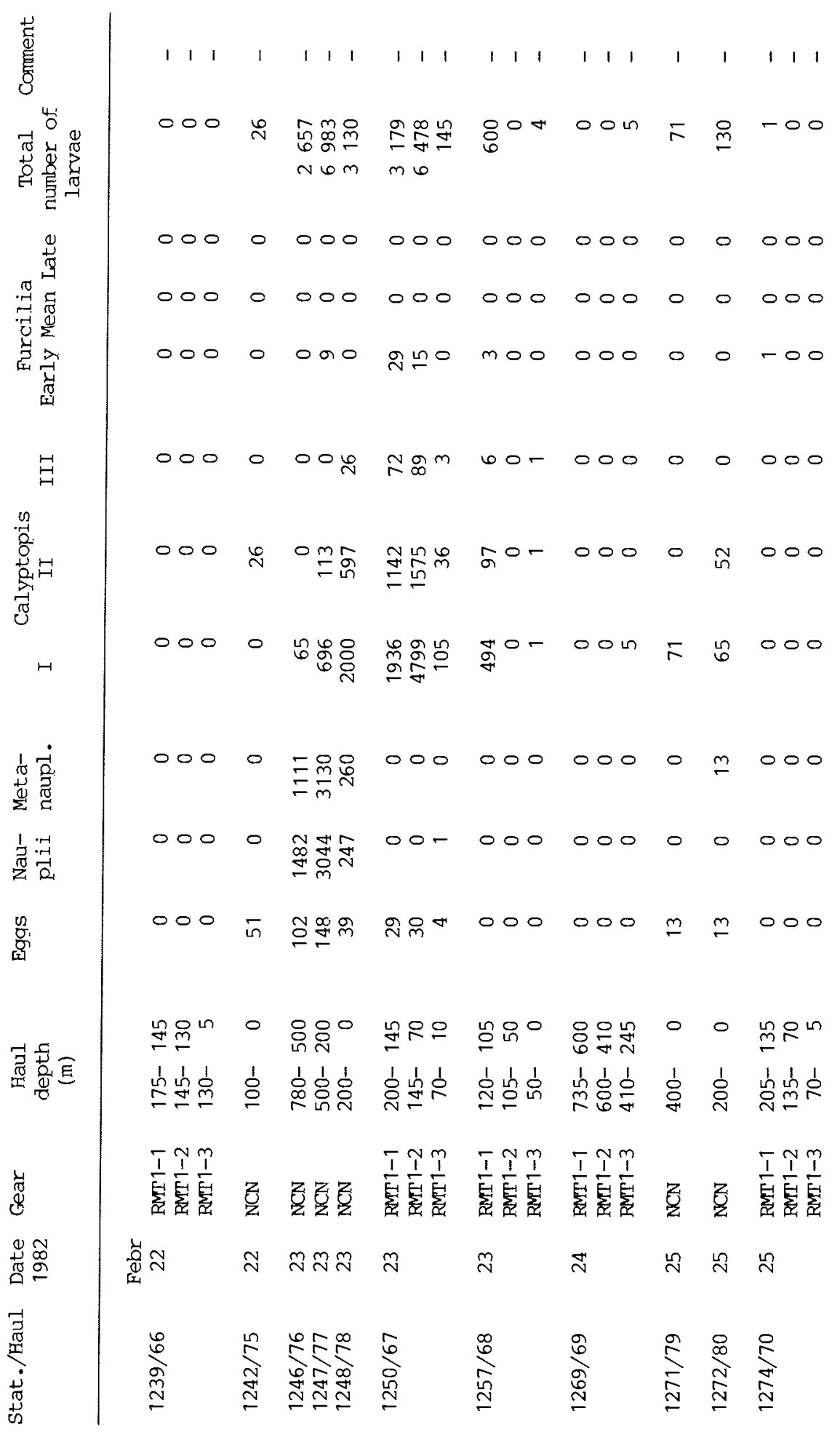




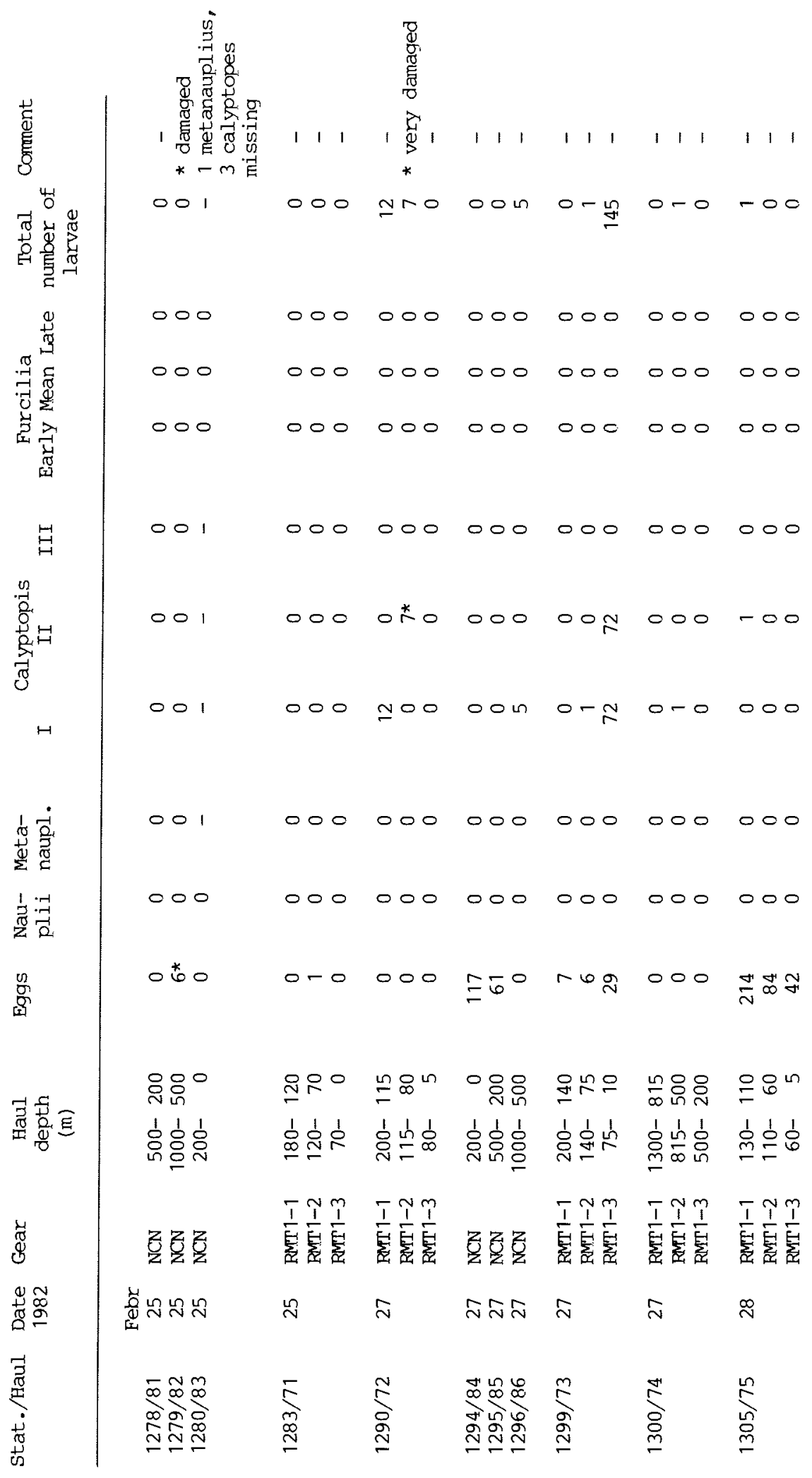




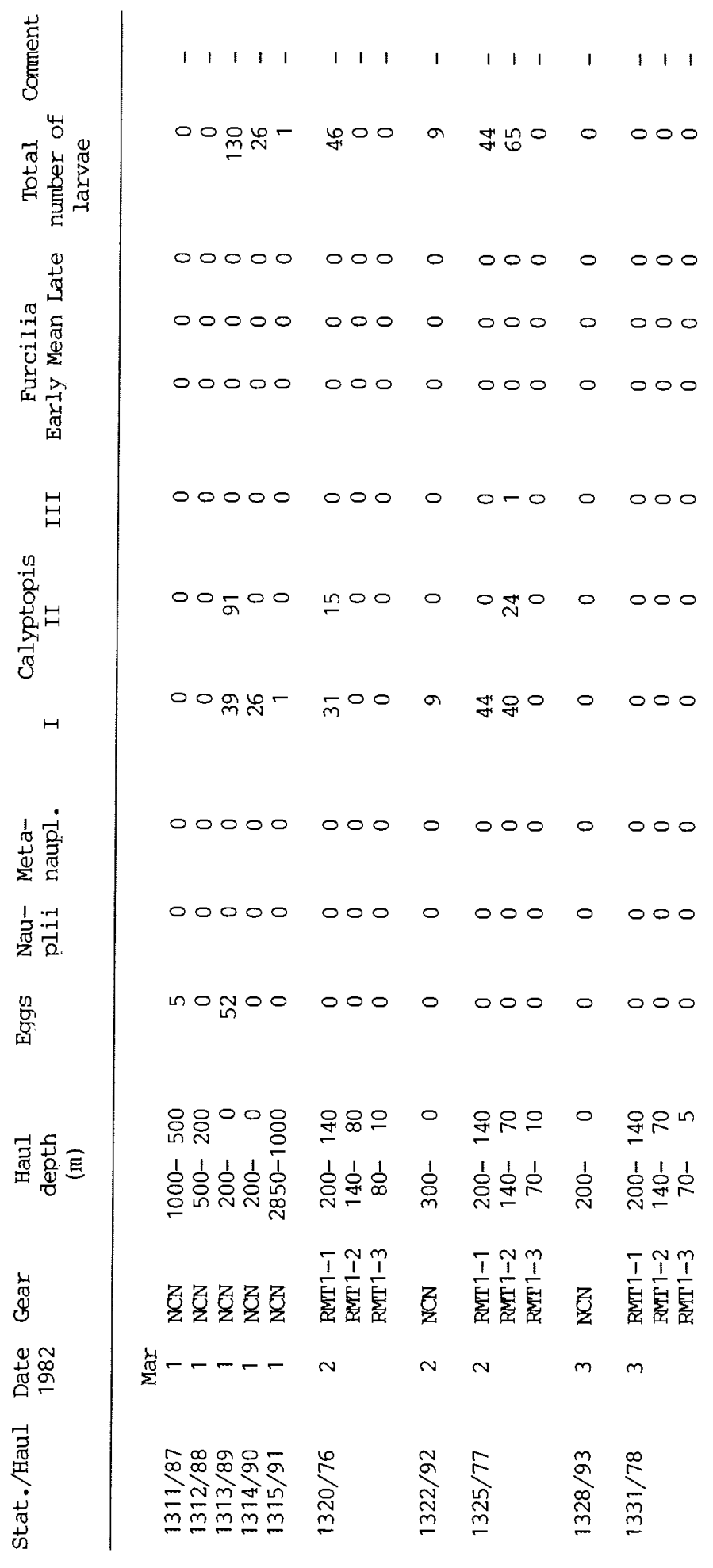


$\underset{N}{\omega}$ Antarctic Expedition 1981/82 RRS "John Biscoe". Distribution of euphausiid larvae other than krill. Used nets: RMT1, NANSEN-CLOSING-NET (NCN). Individuals per $1000 \mathrm{~m}^{3}$.

E. cryst. = Euphausia crystallorophias; E. fr. = Euphausia frigida; $\mathrm{T}$. sp. = Thysanoessa sp.

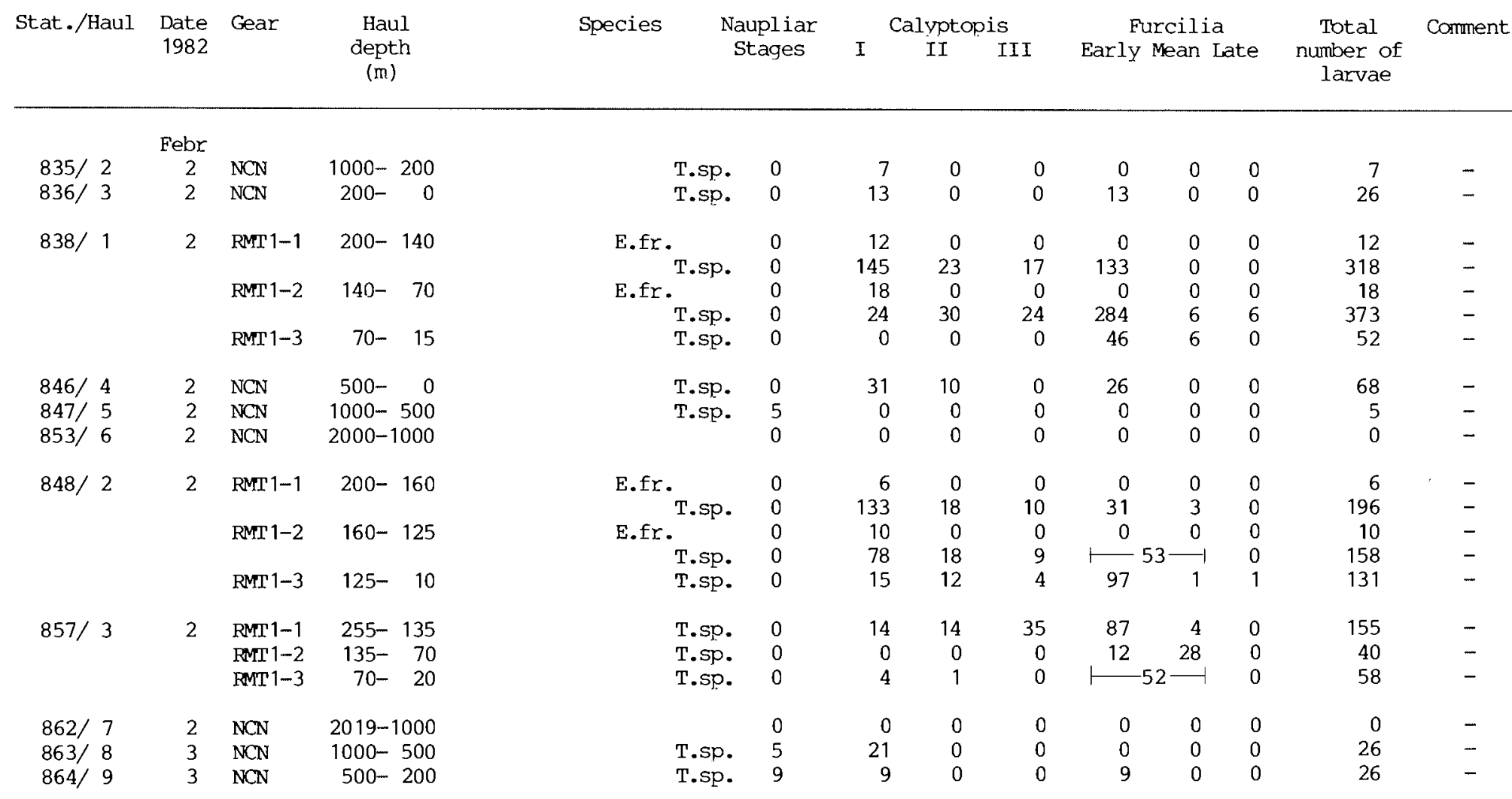




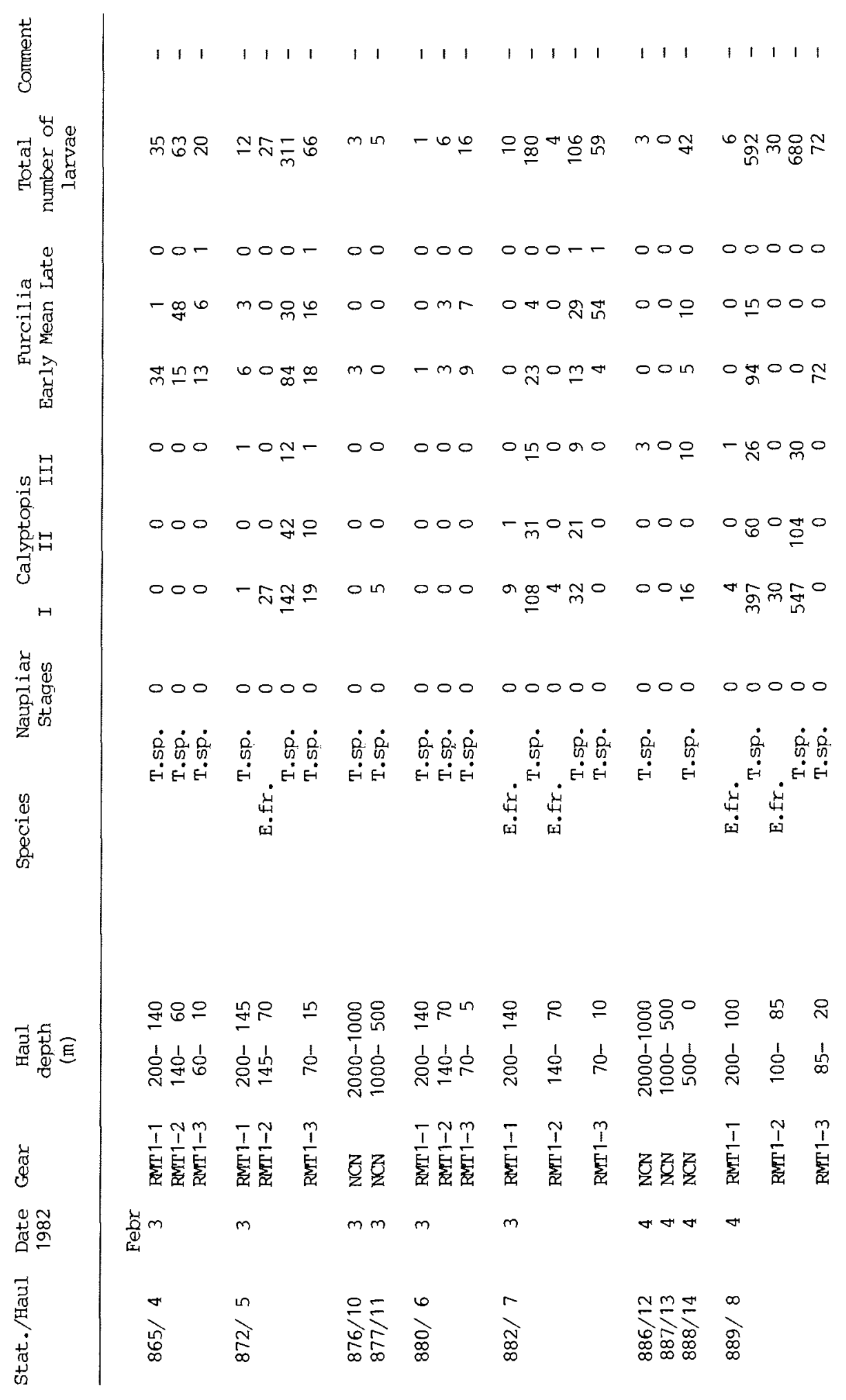




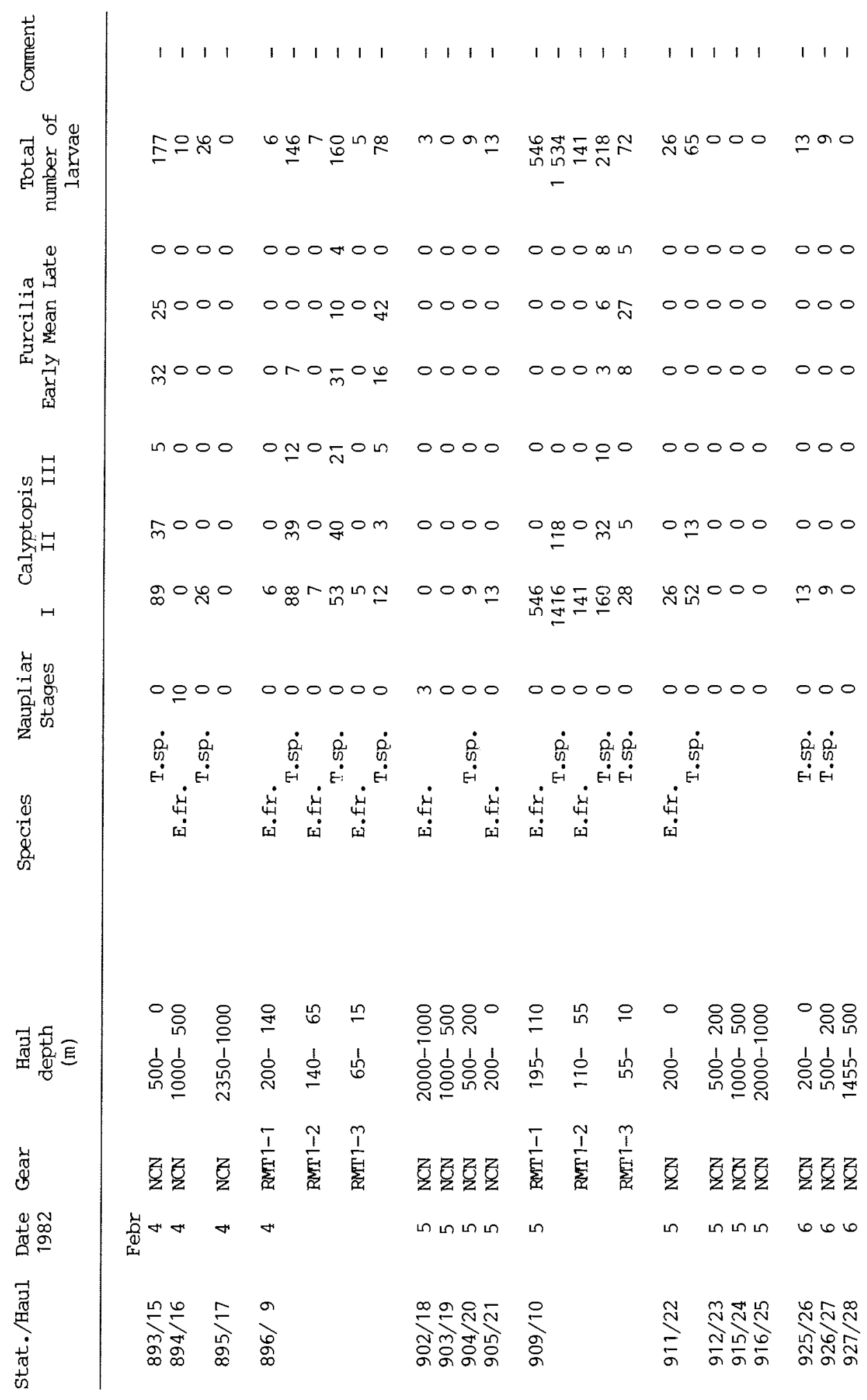




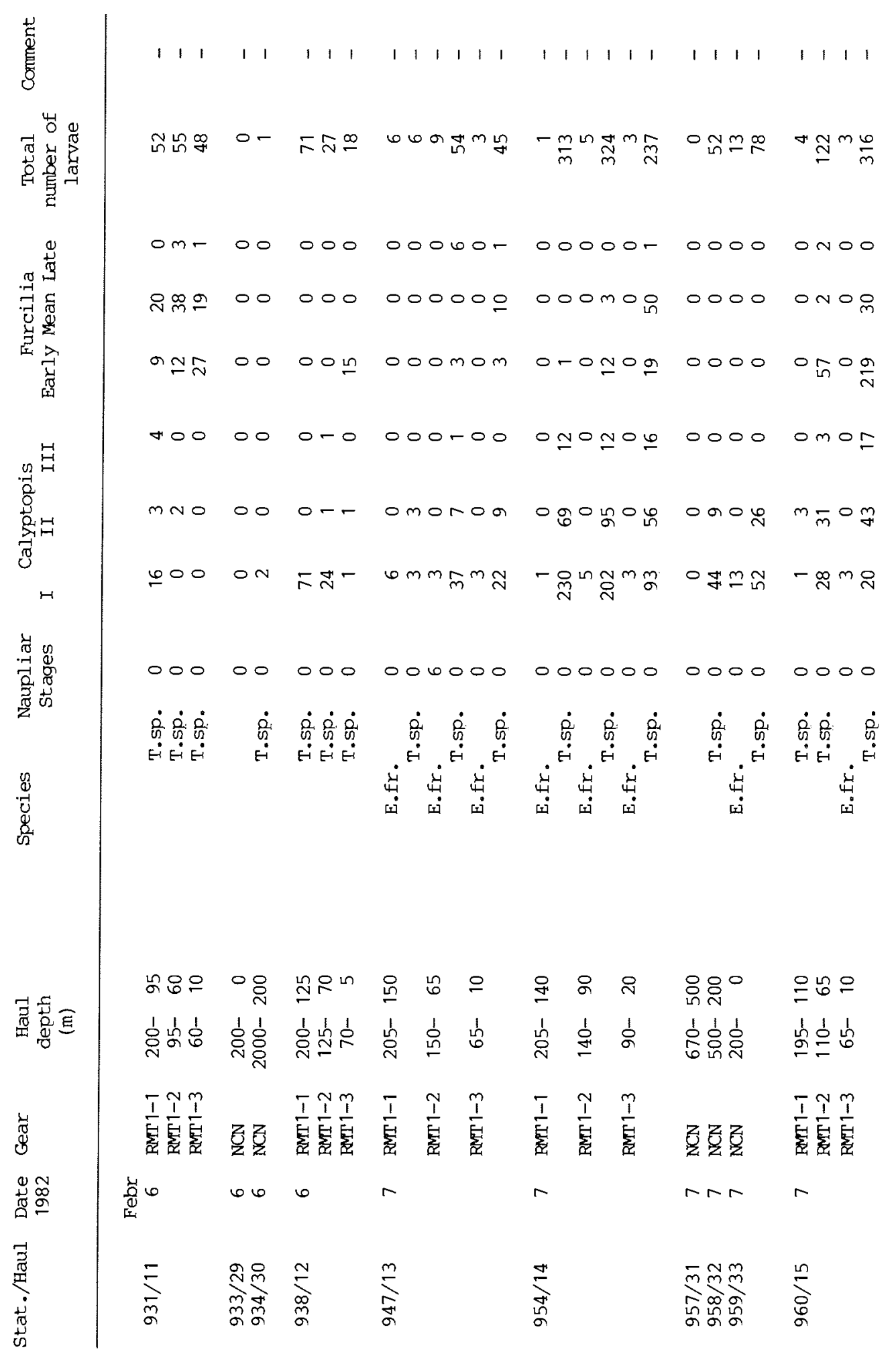




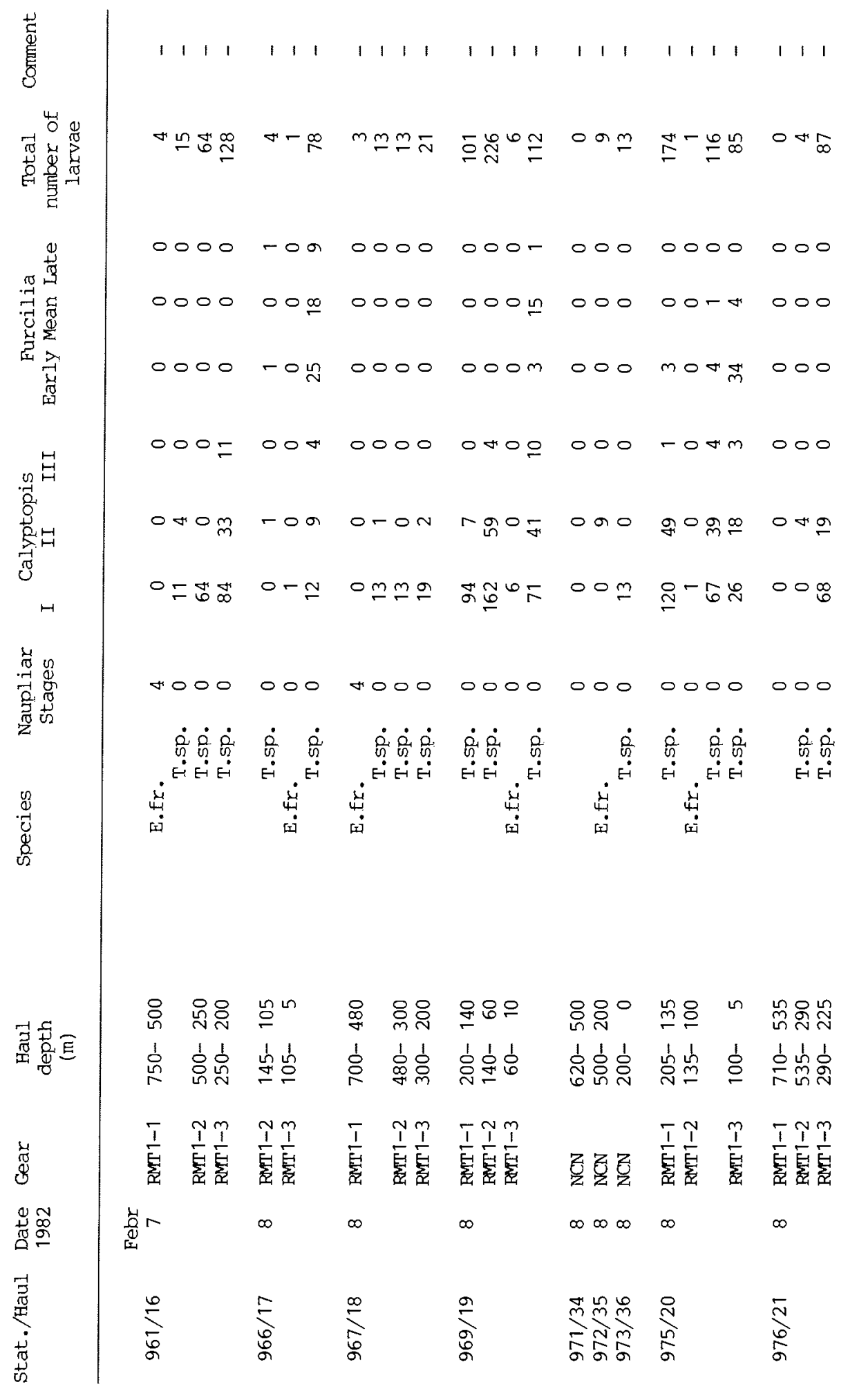




\begin{tabular}{|c|c|c|c|c|c|c|c|c|c|c|c|c|c|}
\hline \multirow[t]{2}{*}{ Stat./Haul } & \multirow{2}{*}{$\begin{array}{l}\text { Date } \\
1982\end{array}$} & \multirow[t]{2}{*}{ Gear } & \multirow{2}{*}{$\begin{array}{l}\text { Haul } \\
\text { depth } \\
\text { (m) }\end{array}$} & \multirow[t]{2}{*}{ Species } & \multirow{2}{*}{$\begin{array}{c}\text { Naupliar } \\
\text { Stages }\end{array}$} & \multicolumn{3}{|c|}{ Calyptopis } & \multicolumn{3}{|c|}{ Furcilia } & \multirow{2}{*}{$\begin{array}{c}\text { Total } \\
\text { number of } \\
\text { larvae }\end{array}$} & \multirow[t]{2}{*}{ Conment } \\
\hline & & & & & & $\mathrm{I}$ & II & III & Early & Mean & Late & & \\
\hline \multirow{4}{*}{$982 / 23$} & Febr & & & & & & & & & & & & \\
\hline & 8 & RMT 1-1 & $185-140$ & T.sp. & 0 & 26 & 6 & 2 & 6 & 9 & 0 & 50 & - \\
\hline & & RMT 1-2 & $140-\quad 60$ & T.sp. & 0 & 40 & 28 & 12 & 12 & 19 & 0 & 111 & - \\
\hline & & RMT1-3 & $60-\quad 10$ & T.SP. & 0 & 13 & 35 & 7 & 165 & 41 & 0 & 262 & - \\
\hline $983 / 37$ & 8 & NCN & $720-500$ & T.sp. & 0 & 0 & 0 & 0 & 12 & 0 & 0 & 12 & - \\
\hline $984 / 38$ & 9 & NCN & $500-200$ & T.sp. & 0 & 9 & 0 & 0 & 0 & 0 & 0 & 9 & - \\
\hline $985 / 39$ & 9 & $\mathrm{NCN}$ & $200-\quad 0$ & T.sp. & 0 & 0 & 26 & 0 & 0 & 0 & 0 & 26 & - \\
\hline \multirow[t]{6}{*}{$986 / 24$} & 9 & RMT 1-1 & $215-140$ & E.fr. & 0 & 8 & 0 & 0 & 8 & 0 & 0 & 15 & - \\
\hline & & & & T.sp. & 0 & 53 & 30 & 8 & 23 & 0 & 0 & 113 & - \\
\hline & & RMT 1-2 & $140-75$ & E.fr. & - & 8 & 0 & 0 & - & - & - & 8 & eggs and fur- \\
\hline & & & & T.sp. & - & 128 & 91 & 23 & - & - & - & 242 & tale \\
\hline & & RMT 1-3 & $75-\quad 5$ & E.fr. & 0 & 6 & 2 & 0 & 0 & 0 & 0 & 8 & - \\
\hline & & $10 \times 1=5$ & (5) & T.sp. & 0 & 3 & 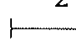 & $46-1$ & 0 & $\longmapsto$ & $-112-$ & 161 & - \\
\hline \multirow[t]{3}{*}{$992 / 25$} & 9 & RMT1-1 & $200-135$ & T.sp. & 0 & 275 & 60 & 9 & 1 & 0 & 0 & 316 & - \\
\hline & & RMT1-2 & $135-60$ & T.sp. & 0 & 38 & 24 & 6 & 16 & 11 & 0 & 95 & - \\
\hline & & RMT1-3 & $60-\quad 10$ & T.sp. & 0 & 0 & 0 & 0 & 26 & 49 & 0 & 75 & - \\
\hline $993 / 40$ & 9 & NCN & $450-200$ & T.sp. & 0 & 10 & 10 & 0 & 0 & 0 & 0 & 21 & - \\
\hline $994 / 41$ & 9 & $\mathrm{NCN}$ & $200-\quad 0$ & T.sp. & 0 & 0 & 26 & 0 & 0 & 13 & 0 & 39 & - \\
\hline \multirow[t]{6}{*}{$997 / 26$} & 9 & RMT1-1 & $200-142$ & E.fr. & 0 & 12 & 0 & 0 & 0 & 0 & 0 & 12 & - \\
\hline & & & & T.sp. & 0 & 20 & 6 & 3 & 0 & 0 & 0 & 29 & - \\
\hline & & RMP1-2 & $142-80$ & E.fr. & 0 & 16 & 0 & 0 & 0 & 0 & 0 & 16 & - \\
\hline & & & & T.sp. & 0 & 1 & 7 & 0 & 0 & 0 & 0 & 9 & - \\
\hline & & FMPT1-3 & $80-20$ & E.fr. & 0 & 14 & 2 & 0 & 0 & 0 & 0 & 15 & - \\
\hline & & & & T.sp. & 0 & 5 & 34 & 8 & $8^{*}$ & 0 & 0 & 54 & * very damagec \\
\hline $1006 / 42$ & 10 & $\mathrm{NCN}$ & $1640-1000$ & & 0 & 0 & 0 & 0 & 0 & 0 & 0 & 0 & - \\
\hline $1007 / 43$ & 10 & $\mathrm{NCN}$ & $1000-500$ & & 0 & 0 & 0 & 0 & 0 & 0 & 0 & 0 & - \\
\hline $1008 / 44$ & 10 & $\mathrm{NCN}$ & $500-200$ & & 0 & 0 & 0 & 0 & 0 & 0 & 0 & 0 & - \\
\hline $1009 / 45$ & 10 & $\mathrm{NCN}$ & $200-\quad 0$ & T.sp. & 0 & 13 & 0 & 0 & 0 & 13 & 0 & 26 & - \\
\hline
\end{tabular}




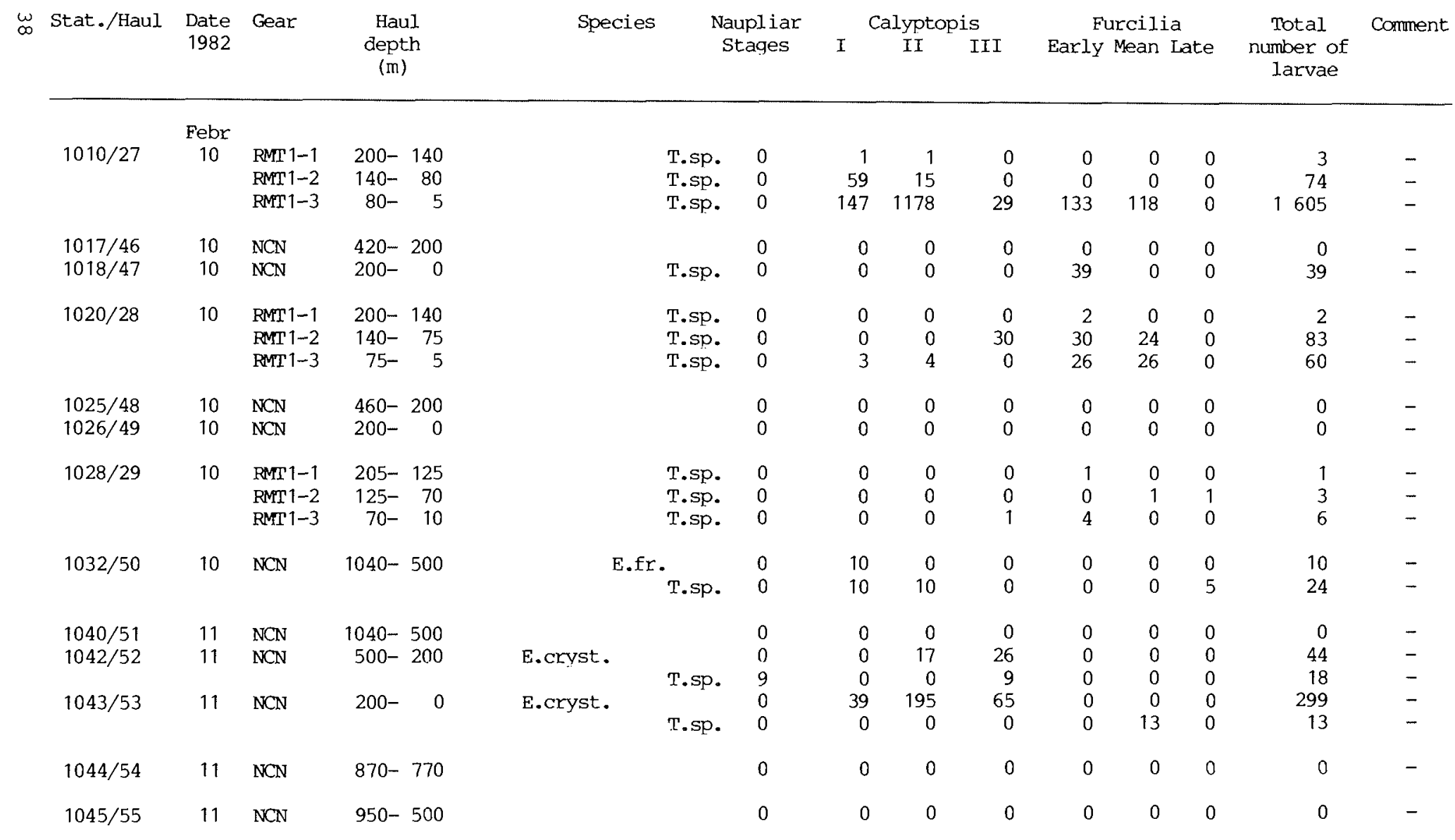




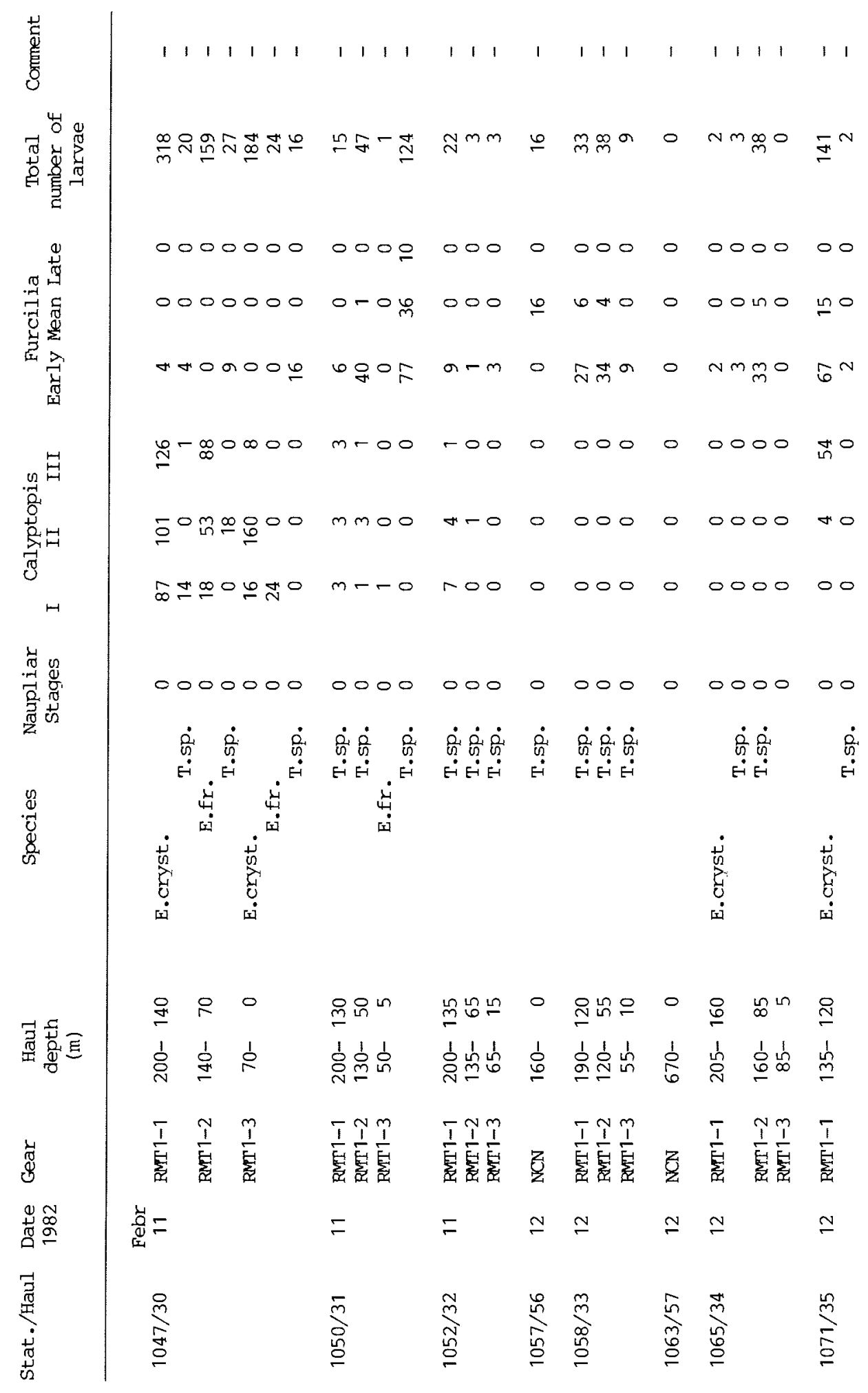




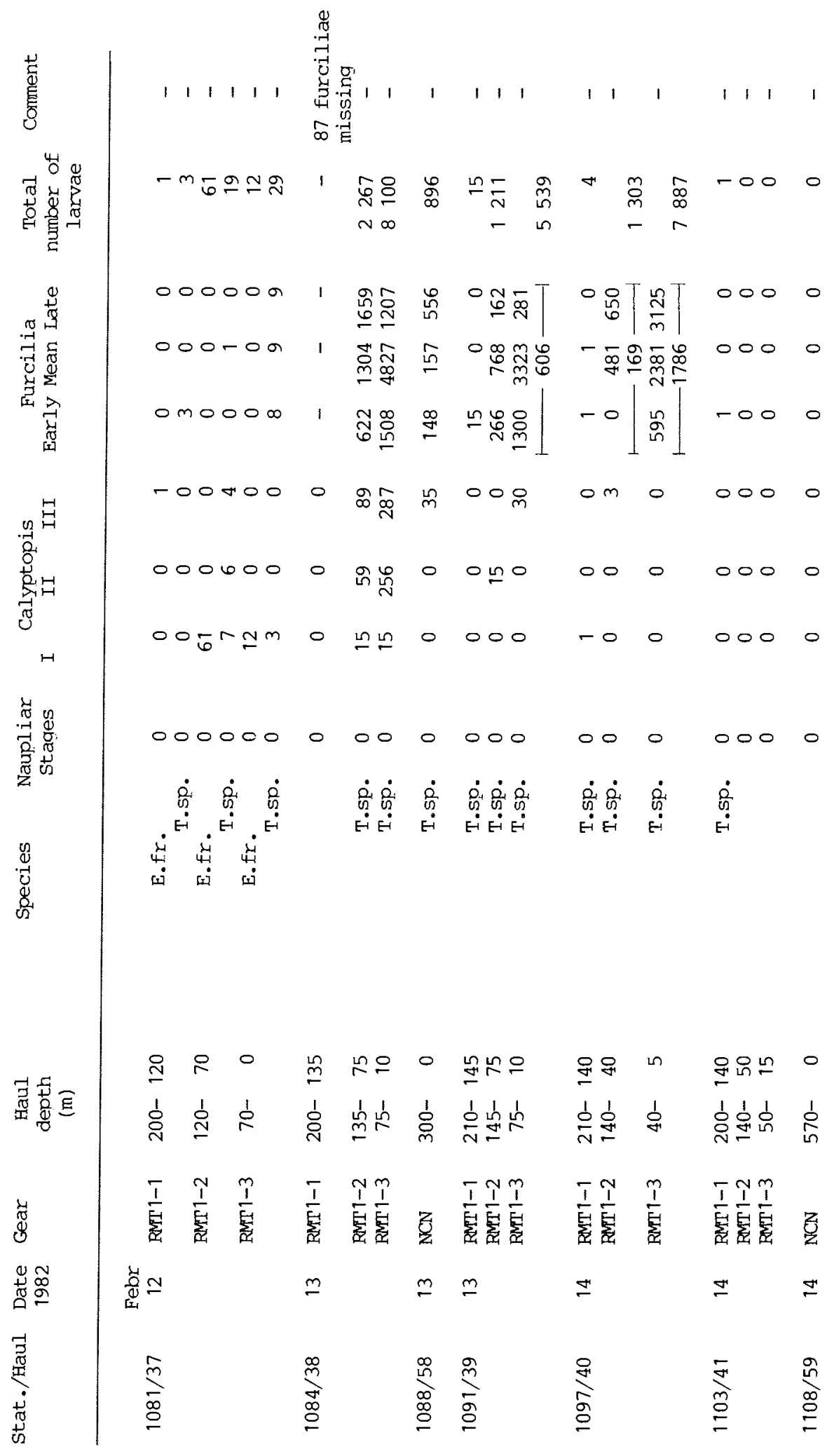




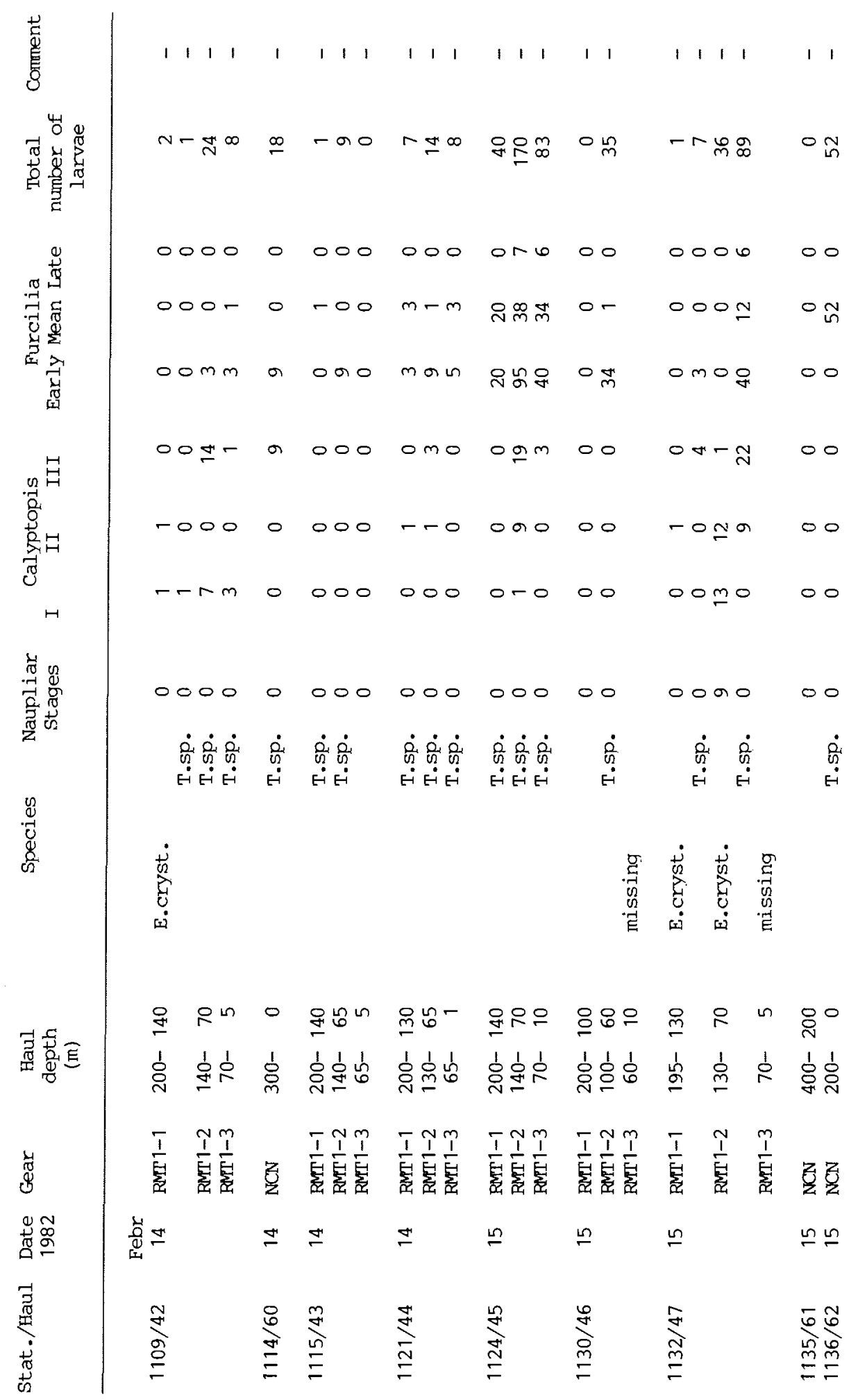




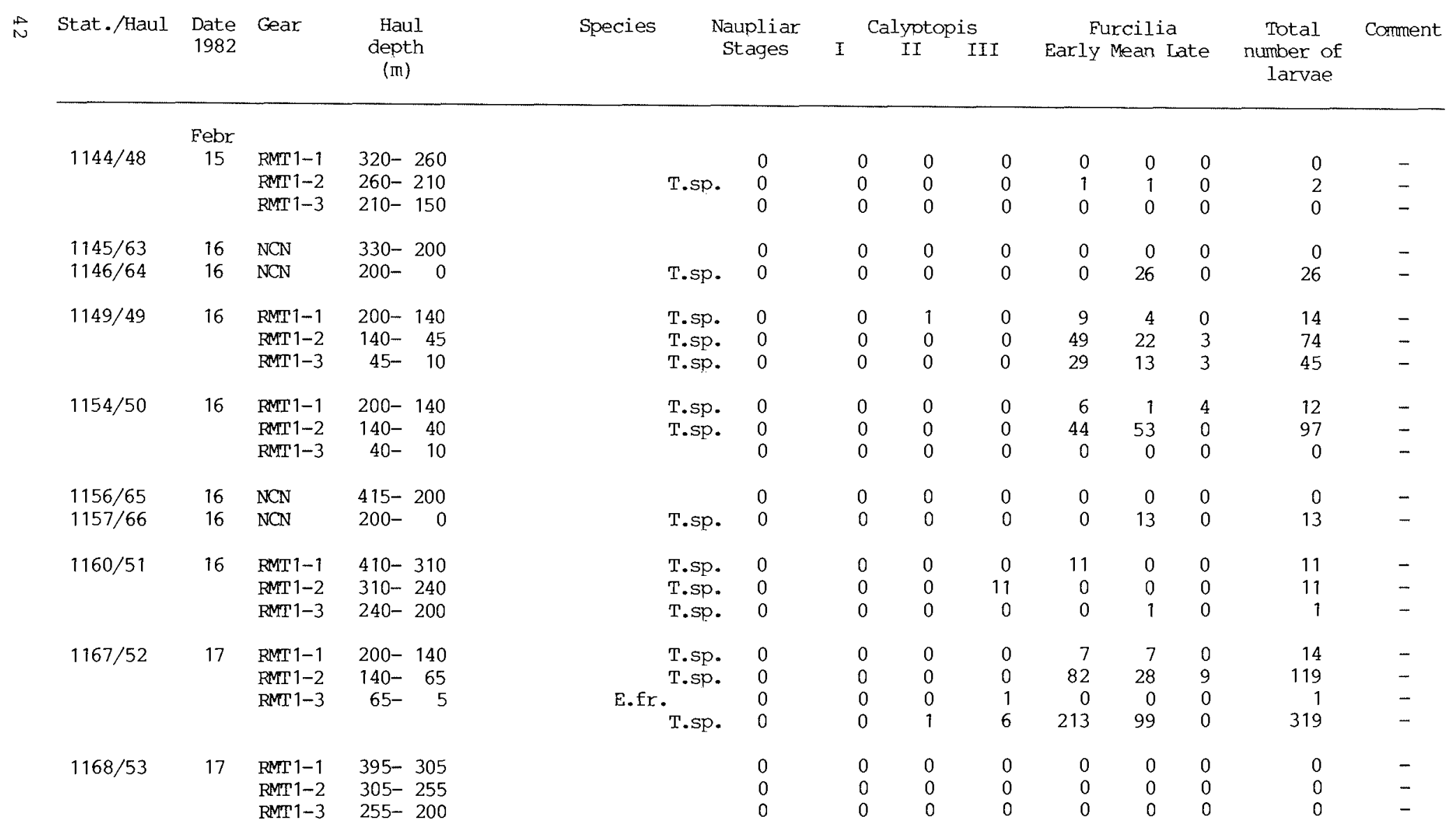




\begin{tabular}{|c|c|c|c|c|c|c|c|c|c|c|c|c|c|c|}
\hline Stat./Haul & $\begin{array}{l}\text { Date } \\
1982\end{array}$ & Gear & $\begin{array}{l}\text { Haul } \\
\text { depth } \\
\text { (m) }\end{array}$ & Species & \multicolumn{2}{|c|}{$\begin{array}{l}\text { Naupliar } \\
\text { Stages }\end{array}$} & \multicolumn{3}{|c|}{ Calyptopis } & \multicolumn{3}{|c|}{ Furcilia } & $\begin{array}{c}\text { Total } \\
\text { number of } \\
\text { larvae }\end{array}$ & Comment \\
\hline \multirow[t]{4}{*}{$1170 / 54$} & 17 & RMT1-1 & $200-140$ & E.cryst. & & 0 & 0 & 15 & 0 & 0 & 0 & 0 & 15 & - \\
\hline & & & & & T.sp. & 0 & 0 & 15 & 29 & 20 & 0 & 0 & 64 & - \\
\hline & & RMT 1-2 & $140-60$ & E. cryst. & & 0 & 4 & 12 & 6 & 0 & 0 & 0 & 22 & - \\
\hline & & & & & T.sp. & 0 & 1 & 6 & 34 & 12 & 3 & 0 & 56 & - \\
\hline $1173 / 67$ & 18 & $\mathrm{NCN}$ & $300-$ & & T.sp. & 0 & 0 & 0 & 0 & 9 & 0 & 0 & 9 & - \\
\hline \multirow[t]{5}{*}{$1174 / 55$} & 18 & RMT1-1 & $200-140$ & & T.sp. & 0 & 0 & 0 & 0 & 1 & 0 & 0 & 1 & - \\
\hline & & RMT 1-2 & $140-70$ & E.cryst. & & 0 & 28 & 6 & 0 & 0 & 0 & 0 & 34 & - \\
\hline & & & & & T.sp. & 0 & 0 & 4 & 6 & 16 & 0 & 0 & 27 & - \\
\hline & & $\operatorname{RMP} 1-3$ & $70-$ & E. cryst. & & 0 & 6 & 3 & 1 & 0 & 0 & 0 & 9 & - \\
\hline & & & & & T.sp. & 0 & 0 & 0 & 2 & 44 & 5 & 0 & 51 & - \\
\hline \multirow[t]{3}{*}{$1183 / 57$} & 18 & RMT1-1 & $200-135$ & & T.sp. & 0 & 3 & 1 & 0 & 0 & 0 & 0 & 4 & - \\
\hline & & RMT1-2 & $135-80$ & & & 0 & 0 & 0 & 0 & 0 & 0 & 0 & 0 & - \\
\hline & & RMT 1-3 & $80-\quad 1$ & & & 0 & 0 & 0 & 0 & 0 & 0 & 0 & 0 & - \\
\hline \multirow[t]{4}{*}{$1187 / 58$} & 19 & RMT 1-1 & $200-150$ & E.cryst. & & 0 & 0 & 0 & 0 & 3 & 0 & 0 & 3 & - \\
\hline & & & & & T.sp. & 0 & 0 & 0 & 1 & 0 & 1 & 0 & 3 & - \\
\hline & & $\operatorname{RMT} 1-2$ & $150-90$ & & T.sp. & 0 & 0 & 0 & 0 & 1 & 0 & 0 & 1 & - \\
\hline & & RMT1-3 & $90-\quad 15$ & & T.sp. & 0 & 0 & 0 & 0 & 3 & 0 & 0 & 3 & - \\
\hline \multirow[t]{3}{*}{$1194 / 59$} & 19 & RMT1-1 & $195-145$ & & & 0 & 0 & 0 & 0 & 0 & 0 & 0 & 0 & - \\
\hline & & RMT 1-2 & $145-70$ & & T.sp. & 0 & 0 & 0 & 0 & 3 & 0 & 0 & 3 & - \\
\hline & & RMT 1-3 & $70-\quad 10$ & & & 0 & 0 & 0 & 0 & 0 & 0 & 0 & 0 & - \\
\hline
\end{tabular}




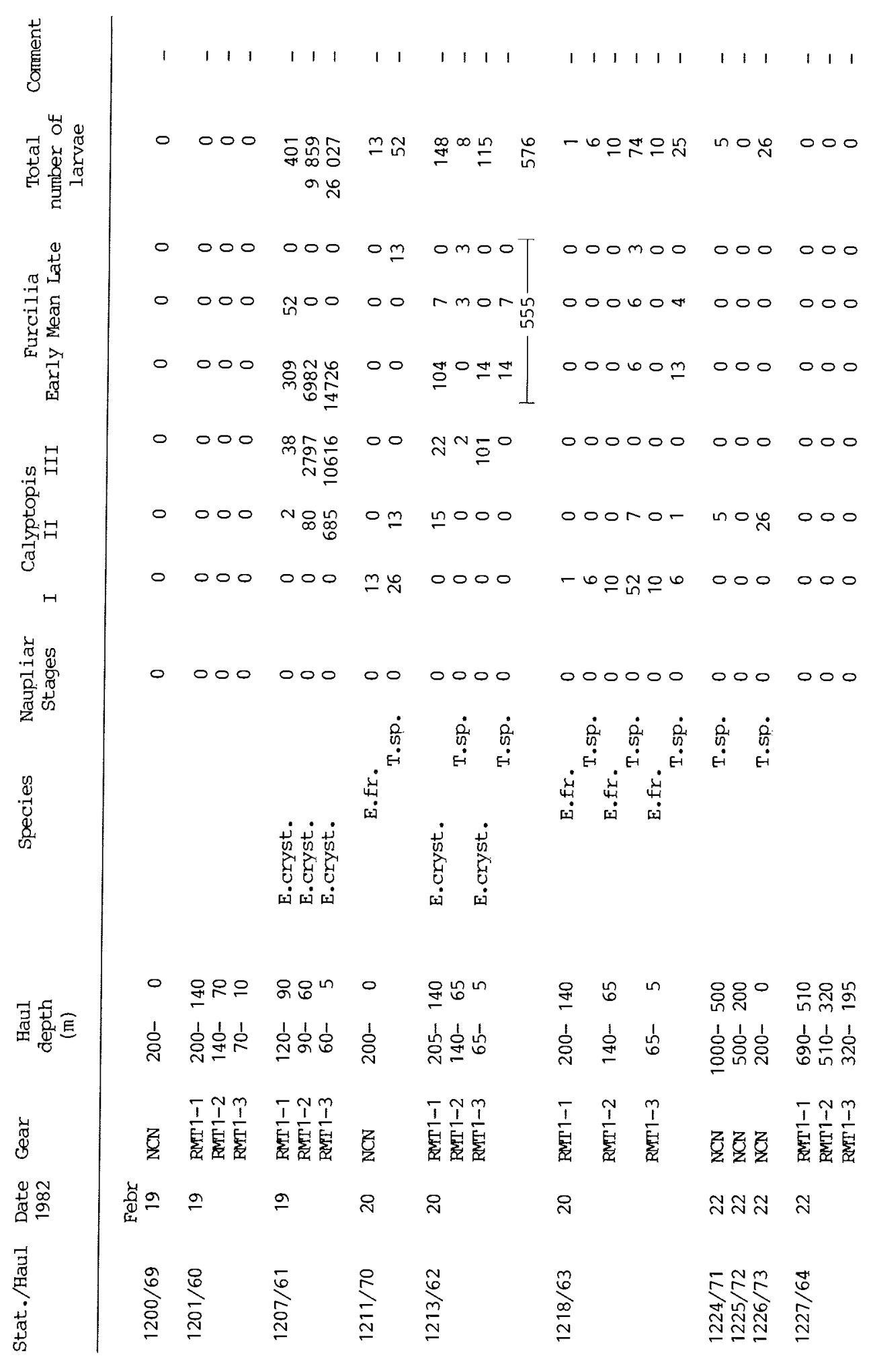




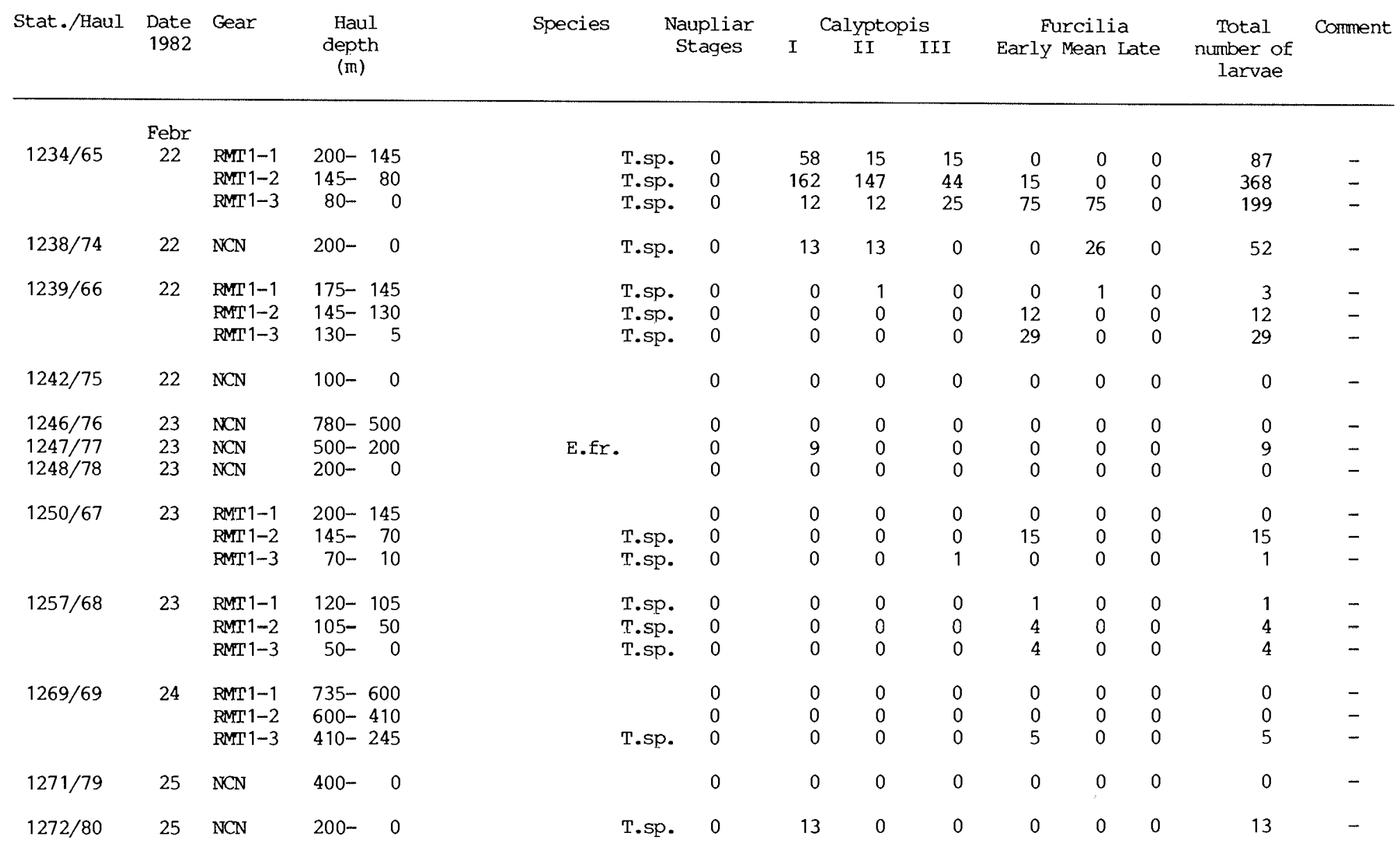




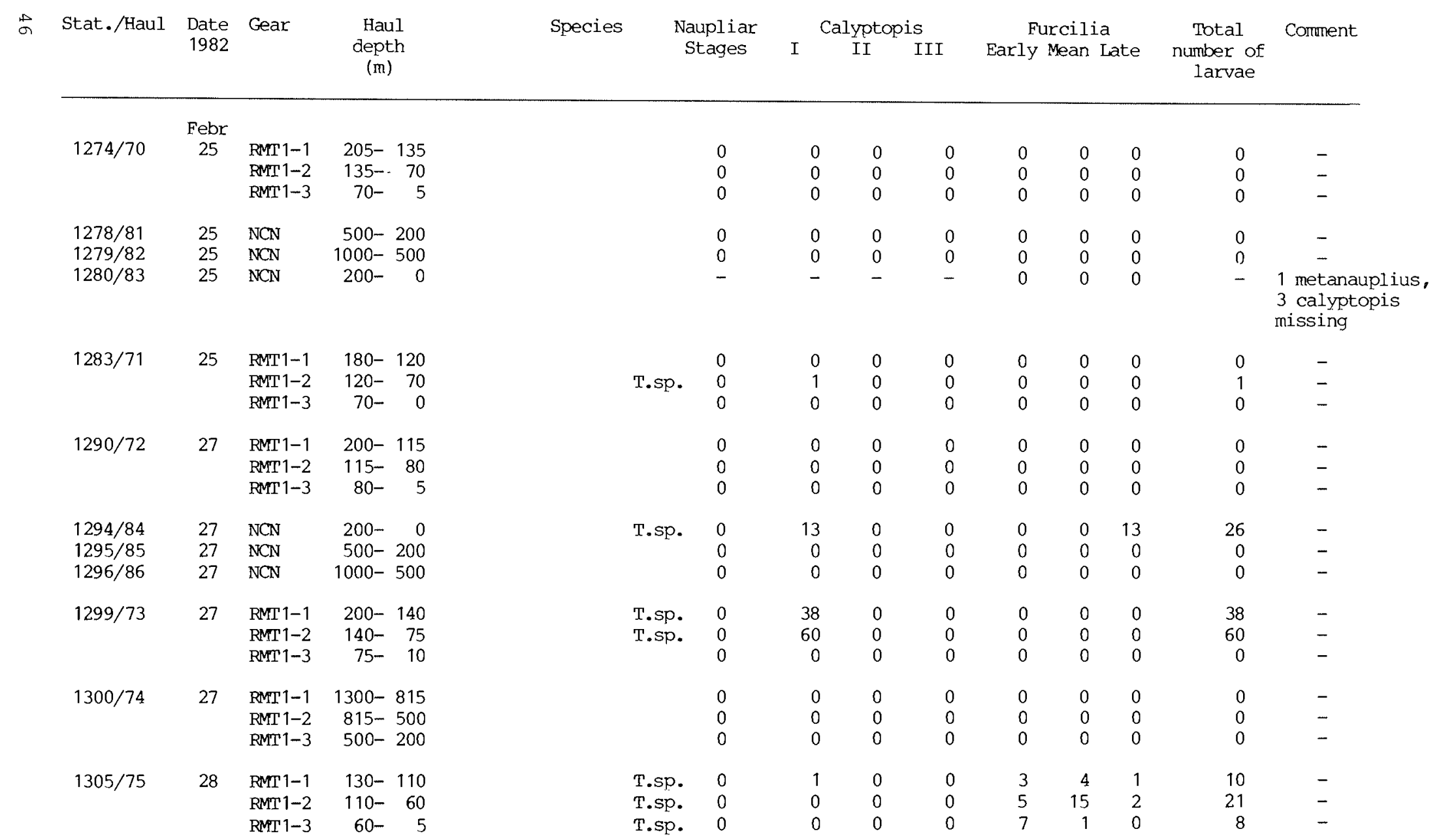




\begin{tabular}{|c|c|c|c|c|c|c|c|c|c|c|c|c|c|}
\hline Stat./Haul & $\begin{array}{l}\text { Date } \\
1981\end{array}$ & Gear & $\begin{array}{l}\text { Haul } \\
\text { depth } \\
\text { (m) }\end{array}$ & Species & $\begin{array}{c}\text { Naupliar } \\
\text { Stages }\end{array}$ & \multicolumn{3}{|c|}{ Calyptopis } & \multicolumn{3}{|c|}{ Furcilia } & $\begin{array}{l}\text { Total } \\
\text { number of } \\
\text { larvae }\end{array}$ & Conment \\
\hline $1311 / 87$ & 1 & $\mathrm{NCN}$ & $1000-500$ & & 0 & 0 & 0 & 0 & 0 & 0 & 0 & 0 & - \\
\hline $1312 / 88$ & 1 & NCN & $500-200$ & E.fr. & 9 & 17 & 0 & 0 & 0 & 0 & 0 & 26 & - \\
\hline \multirow[t]{2}{*}{$1313 / 89$} & 1 & $\mathrm{NCN}$ & $200-\quad 0$ & E.fr. & 0 & 65 & 13 & 0 & 0 & 0 & 0 & 78 & _- \\
\hline & & & & T.sp. & 0 & 0 & 13 & 0 & 0 & 0 & 0 & 13 & - \\
\hline $1315 / 91$ & 1 & NCN & $2850-1000$ & & 0 & 0 & 0 & 0 & 0 & 0 & 0 & 0 & - \\
\hline \multirow[t]{6}{*}{$1320 / 76$} & 2 & RMT1-1 & $200-140$ & E.fr. & 0 & 260 & 31 & 0 & 0 & 0 & 0 & 290 & _- \\
\hline & & & & T.sp. & 0 & 260 & 183 & 15 & 0 & 15 & 0 & 473 & - \\
\hline & & RMT1-2 & $140-80$ & E.fr. & 0 & 295 & 44 & 0 & 0 & 0 & 0 & 339 & - \\
\hline & & & & T.sp. & 0 & 1591 & 928 & 74 & 0 & 118 & 0 & 2725 & - \\
\hline & & RMP1-3 & $80-\quad 10$ & E.fr. & 0 & 14 & 0 & 0 & 0 & 0 & 0 & 14 & - \\
\hline & & & & T.sp & 0 & 0 & 58 & 29 & 29 & 29 & 58 & 203 & - \\
\hline \multirow{4}{*}{$1325 / 77$} & & RMT 1-2 & $140-70$ & E.fr. & 0 & 41 & 24 & 15 & 0 & 0 & 0 & 80 & - \\
\hline & & & & T.sp. & 0 & 25 & 12 & 9 & 130 & 221 & 65 & & - \\
\hline & & RMP1-3 & $70-10$ & E.fr. & 0 & 45 & 166 & 60 & 0 & $\begin{array}{r}86 \\
0\end{array}$ & $\overline{0}$ & $\begin{array}{l}54 / \\
271\end{array}$ & - \\
\hline & & & & T.sp. & 0 & 60 & 211 & 45 & 2259 & 2560 & 1355 & & - \\
\hline $1328 / 93$ & 3 & $\mathrm{NCN}$ & $200-\quad 0$ & & 0 & 0 & 0 & 0 & 0 & 0 & 0 & 0 & - \\
\hline \multirow[t]{3}{*}{$1331 / 78$} & 3 & RMT1-1 & $200-140$ & & 0 & 0 & 0 & 0 & 0 & 0 & 0 & 0 & - \\
\hline & & RMT $1-2$ & $140-70$ & T.sp. & 0 & 0 & 0 & 0 & 0 & 15 & 147 & 162 & - \\
\hline & & RMT $1-3$ & $70-5$ & T.sp. & 0 & 0 & 0 & 0 & 15 & 208 & 565 & 789 & - \\
\hline
\end{tabular}


